\title{
Cancer-associated mRNAs regulated by the Helix-Loop-Helix motif of human EIF3A
}

Short title: Human mRNAs regulated by the HLH motif in EIF3A

Marina Volegova ${ }^{1}$ and Jamie H.D. Cate ${ }^{1,2,3 *}$

${ }^{1}$ Department of Molecular and Cell Biology, University of California, Berkeley, CA

${ }^{2}$ Physical Biosciences Division, Lawrence Berkeley National Laboratory, Berkeley, CA

${ }^{3}$ Department of Chemistry, University of California, Berkeley, CA

Correspondence to: Jamie H.D. Cate (jcate@lbl.gov) 


\section{Abstract}

Improper regulation of translation initiation, a vital check-point of protein synthesis in the cell, has been linked to a number of cancers. Overexpression of protein subunits of eukaryotic translation initiation factor 3 (eIF3) has been associated with increased translation of mRNAs involved in cell proliferation. In addition to playing a major role in general translation initiation by serving as a scaffold for the assembly of translation initiation complexes, eIF3 regulates translation of specific cellular mRNAs and viral RNAs. Mutations in the N-terminal Helix-LoopHelix (HLH) RNA-binding motif of the EIF3A subunit in eIF3 interfere with Hepatitis C Virus Internal Ribosome Entry Site (IRES) mediated translation initiation in vitro. Here we show that the EIF3A HLH motif controls translation of a small set of cellular transcripts enriched in oncogenic mRNAs, including $M Y C$. We also demonstrate that the HLH motif of EIF3A acts specifically on the 5'-UTR of MYC mRNA and modulates the function of EIF4A1 on select transcripts during translation initiation. In Ramos lymphoma cell lines, which are dependent on MYC overexpression, mutations in the HLH motif greatly reduce MYC expression, impede proliferation and sensitize cells to anti-cancer compounds. These results reveal the potential of the EIF3A HLH motif in eIF3 as a promising chemotherapeutic target.

\section{Summary}

The Helix Loop Helix motif of EIF3A controls translation of a small set of oncogenic cellular transcripts, including $M Y C$, and modulates the function of translation initiation factor EIF4A1 during translation initiation on select mRNAs. 


\section{Introduction}

Eukaryotic translation initiation is tightly controlled and its deregulation can lead to a wide variety of disorders, including cancer (1). During canonical translation initiation, the eukaryotic small (40S) ribosomal subunit first associates with initiation factors eIF1, eIF1A, eIF3, and eIF5, and is subsequently loaded with the eIF2 ternary complex (eIF2-GTP-Met-tRNA ${ }_{i}^{\text {Met }}$ ). It then binds to the mRNA-bearing eIF4F complex (EIF4A, 4B, 4G, 4E) to begin directional scanning of the $5^{\prime}$ untranslated region (5'-UTR) for the start codon (2). Distinct from the canonical scanning mechanism, recent evidence indicates that eIF3-the largest of the translation initiation factors comprised of 13 subunits (EIF3A-M) in mammals-regulates alternative pathways of translation initiation. For select cellular mRNAs, eIF3 can either activate or repress translation by interacting with RNA structural elements in the 5'-UTRs of these mRNAs (3). Additionally, eIF3 can bind the 5'-cap of mRNAs using EIF3D (4), allowing translation of select transcripts to continue under cellular stress conditions when eIF4E is inactivated. Finally, eIF3 can also promote translation under stress conditions by binding $\mathrm{m}^{6} \mathrm{~A}$-methylated 5 '-UTRs in a cap-independent manner $(5,6)$. In all these cases, the molecular basis for eIF3-dependent translation regulation and its control of gene expression networks remain unclear.

Structural analysis using cryo-electron microscopy (cryo-EM) revealed that the core of eIF3, a five-lobed octameric complex, localizes to the solvent-exposed "backside" of the $40 \mathrm{~S}$ subunit and spans the mRNA entry and exit channels (7). In the mammalian 43S pre-initiation complex (PIC), eIF3 subunits EIF3A and EIF3C directly contact the 40S subunit (8), as well as participate in interactions with eIF1, eIF1A, eIF2, eIF5, and eIF4F $(9,10)$, thus coordinating the ordered assembly of the $48 \mathrm{~S}$ initiation complex. By contrast with canonical initiation, translation of the Hepatitis C Virus (HCV) genomic RNA requires an Internal Ribosome Entry Site (HCV IRES) RNA structure in the 5'-UTR, which binds to subunits EIF3A and EIF3C within the eIF3 
complex (11). Recent cryo-EM structures revealed how an HCV-like viral IRES displaces eIF3 from binding the 40S subunit, while still binding to eIF3 through subunits EIF3A and EIF3C (12) (Fig. 1A). The importance of the EIF3A subunit in mediating RNA binding is underscored by in vitro translation (IVT) experiments in which mutation of amino acids 36-39 (KSKK > NSEE) in a predicted RNA-binding HLH ( (11). While these experiments indicated that the HLH motif of EIF3A is critical for mediating HCV IRES binding by eIF3, the importance of this motif for cellular mRNA translation remains to be determined.

Beyond the role of EIF3D in binding the mRNA $\mathrm{m}^{7} \mathrm{G}$ cap (4), the mechanisms responsible for eIF3-mediated regulation of specific cellular mRNAs remain unclear. Structural models for eIF3 bound to $43 \mathrm{~S}$ and $48 \mathrm{~S}$ pre-initiation complexes suggest that eIF3 controls the translation of the HCV IRES in a distinct manner compared to cellular transcripts, whether involving canonical scanning or eIF3-dependent regulation of specific transcripts. In addition to its displacement from the 40S subunit by the HCV IRES RNA in the cryo-EM reconstruction, the HLH motif in EIF3A is spatially distant from the EIF3D cap-binding domain (10) and is more discrete than the proposed multi-subunit interface thought to recognize specific RNA secondary structures (3) and $\mathrm{m}^{6} \mathrm{~A}$ modifications $(5,13)$. We therefore probed the role of the EIF3A HLH motif in regulating cellular translation initiation in cells and in vitro. We found that mutations in the EIF3A HLH motif affected the translational efficiency of transcripts involved in proliferative pathways, including the mRNAs encoding MYC, PRL3 and MET. MYC is a well-known transcription factor strongly associated with cancer initiation and is found to be deregulated in over half of human cancers, whereas PRL3 and MET are implicated in cancer metastasis through regulating oncogenic effector pathways, such as PI3K/Akt/mTOR and HGF/SF signaling, respectively (1416). The selective enhancement of translation initiation on cancer-associated transcripts by the 
EIF3A HLH motif highlights a new mode of eIF3 translation regulation and identifies a welldefined, discrete structural motif (13) that could be targeted for future drug development efforts.

\section{Results}

Mutation of the EIF3A HLH motif causes selective translation repression of proliferative mRNAs

We first introduced a 3 amino-acid mutation in the HLH RNA-binding motif in EIF3A $($ KSKK > NSEE, hereafter termed EIF3A HLH*), previously shown to disrupt HCV IRESmediated translation initiation (11), into HEK293T cells using a lentiviral delivery and integration system under hygromycin selection. We then knocked down endogenous EIF3A expression using an shRNA targeting the native mRNA 3'-UTR, expressed by a second lentiviral system under dual hygromycin/puromycin selection (table S1). We also generated control (CT) cell lines in the same manner but with no mutation in the exogenous EIF3A sequence. HLH* cell lysates showed a dramatic $(\sim 80 \%)$ decrease in encephalomyocarditis virus (EMCV) IRES-mediated translation, consistent with the effects previously seen in vitro with reconstituted eIF3 and the HCV IRES

(Fig. 1B) (11).

We then used the CT and HLH* cell lines to determine the effect of the EIF3A HLH mutation on the translational efficiency of cellular mRNAs. To assess the extent of mutant EIF3A expression, RNA deep sequencing (RNAseq) data were aligned to wild-type and HLH* EIF3A sequences, revealing that $>80 \%$ of aligned $\mathrm{HLH}$ reads mapped to the $\mathrm{HLH}^{*}$ sequence, and confirming robust expression of exogenous over endogenous EIF3A (Fig. 1C). At the transcriptional level, HLH* EIF3A had little effect on mRNA expression (fig. S1). However, bioinformatic analysis of ribosome profiling data, normalized to the RNAseq data, revealed a small number of transcripts differentially regulated at the translational level in cells expressing 


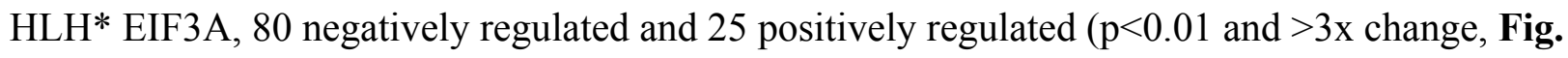
1D, fig. S2A). Functional classification of these genes showed strong enrichment of oncogenic transcripts (Fig. 1E), including those encoding PRL3, MYC and MET, all of which were negatively affected by HLH* EIF3A (Fig. 1D). Consistent with the ribosome profiling results, Western blot analysis showed a decrease in protein levels for MYC, MET, and PRL3 (Fig. 1F). Notably, in experiments using transient expression of HLH* EIF3A, MYC protein levels were also suppressed at 72 hours post-transfection, when transcript levels for the mRNA encoding

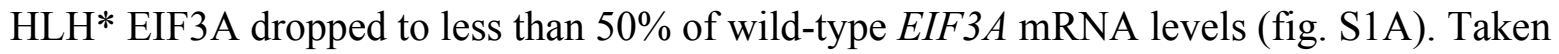
together, these results indicate that HLH* EIF3A lowers the translation of certain oncogenic transcripts such as $M Y C$ that are important for cell proliferation, in a dominant negative manner.

In longer timeframes than those used to collect samples for the ribosome profiling and RNAseq experiments, we observed that the shRNA cell lines expressing HLH* EIF3A had reverted MYC protein expression to those seen in CT cells, and proliferated like CT cells with increased passage number. We therefore engineered HEK293T cells to express CT or HLH* EIF3A from transducted lentiviral vectors, as in the shRNA cell lines, but instead using CRISPRi to suppress expression of endogenous EIF3A (table S1). In the CRISPRi cell lines, we observed the same decrease in MYC, MET, and PRL3 levels by Western blot as in the shRNA cell lines (Fig. 1G). Although the CRISPRi cell lines exhibited a moderate global decrease in translation, in contrast to the shRNA-based cell lines as determined by metabolic labeling (fig. S1B), we continued to use the CRISPRi cell lines for subsequent biochemical and luciferase reporter-based experiments since they were more stable with passage number compared to the shRNA cell lines, as assessed by growth rate and relative MYC levels as a function of passage number. 
To test the effect of HLH* EIF3A on translation initiation, we used the CRISPRi-based CT and HLH* cell lines to prepare cytoplasmic extracts for in vitro translation experiments. We programmed these extracts with full-length $G A P D H$ and $M Y C$ mRNAs, and stalled translation reactions with either cycloheximide or GMPPNP, and fractionated them on sucrose gradients (Fig. 2, A and B). Cycloheximide stalls $80 \mathrm{~S}$ ribosomes immediately after initiation, whereas GMPPNP stalls 48S pre-initiation complexes at the start codon $(11,17)$. In Western blots of the sucrose gradient fractions, we observed a defect in EIF3A and EIF5B distribution in the HLH* EIF3A in vitro translation reactions programmed with either $G A P D H$ or $M Y C$ mRNA (Fig. 2, C F). These results are consistent with those using reconstituted eIF3 and the HCV IRES, which showed that the EIF3A HLH motif is important for eIF3 association with the 40S subunit, and for EIF5B association with pre-initiation complexes and the $80 \mathrm{~S}$ ribosome (11), and suggest these defects are general. We did not observe a defect in the distribution of Met-tRNA $\mathrm{i}_{\mathrm{i}}$ contrast to the

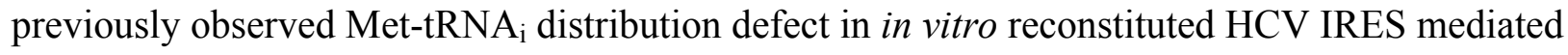
translation with reconstituted eIF3 (11) (fig. S3A-C). Notably, in vitro translation reactions using

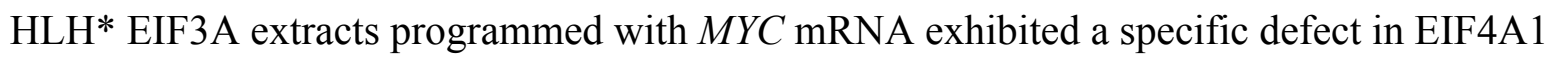
incorporation into preinitiation complexes (Fig. 2, E and F). The defect in EIF4A1 distribution occurred in both cycloheximide and GMPPNP stalled reactions, suggesting that HLH* EIF3A selectively destabilizes $48 \mathrm{~S}$ pre-initiation complexes on some mRNAs (Fig. 2, fig. S4A).

\section{HLH*EIF3A sensitizes translation extracts to eIF4A1 inhibitor RocA}

To address the destabilizing effect of HLH* EIF3A on EIF4A1 incorporation into 48S pre-initiation complexes, we used Rocaglamide A (RocA) to inhibit EIF4A1 function in CT and

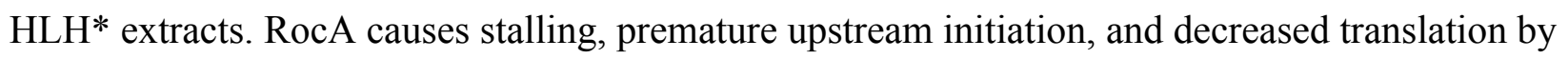
locking EIF4A1 onto poly-purine sequences in the 5'-UTR of mRNAs (18). In vitro translation of Renilla luciferase reporter mRNAs harboring the HCV IRES or MYC 5'-UTR showed a marked 
translation defect in extracts from the HLH* cells (Fig. 3A). By contrast, a Firefly luciferase reporter mRNA with the $H B B$ 5'-UTR used as a control was unaffected by HLH* EIF3A, indicating that the $\mathrm{HLH}^{*}$ mutation is specific to EIF3A HLH-sensitive mRNAs in a 5'-UTR dependent manner (Fig. 3A). Notably, HLH* EIF3A further sensitizes the HCV IRES and the MYC 5'-UTR to RocA in the in vitro translation reactions (Fig. 3B). The RocA-dependent decrease in translation occurred in addition to the HLH*-specific defect (Fig. 3A). Importantly, neither of these mRNAs were sensitive to RocA in the CT lysate, consistent with previous results (18).

To assess the effect of RocA on translation pre-initiation complex formation, we used in vitro translation reactions programmed with full-length $G A P D H$ or $M Y C$ mRNAs and inhibited the reactions with both GMPPNP and RocA. Western blots of fractionated reactions showed a further decrease in EIF4A1 recruitment in the $\mathrm{HLH}^{*}$ in vitro translation extracts programmed with MYC mRNA compared to GMPPNP alone (Fig. 3, C - E, compare to fig. S4, A and B). RocA did not affect EIF4A1 distributions in GAPDH-programmed reactions or in CT extracts programmed with $M Y C$ mRNA, showing that the combined defect of the HLH* EIF3A and RocA on EIF4A1 association with $48 \mathrm{~S}$ pre-initiation complexes is also transcript-specific. No significant difference was observed for Met-tRNA $\mathrm{A}_{\mathrm{i}}$ distribution, suggesting it is not perturbed by RocA (fig. S4C).

\section{EIF3A HLH motif interacts with mRNAs in counterpoint to EIF4A1}

In the yeast Saccharomyces cerevisiae, the EIF3A N-terminal domain (NTD), which includes the HLH motif, has been shown to enhance re-initiation upon translation of upstream open reading frames uORF1 and uORF2 of GCN4 (19-21), likely by interacting with mRNA at the mRNA exit channel of the 40S subunit (22). Notably, in addition to being tuned to levels of 
active eIF2, control of GCN4 translation by the uORFs in its 5'-UTR also requires scanning in an eIF4A-dependent manner (23). Translational control of the functional ortholog of Gen4 in mammals - stress response transcription factor ATF4 - also requires uORFs (24). Briefly, after translation of ATF4 uORF1 under normal conditions, ribosomes re-initiate and continue scanning, encountering inhibitory uORF2, which causes dissociation before the start codon (24). Under stressed conditions with low eIF2, a portion of ribosomes scans through uORF2 and initiates at the start codon. Deletion of uORF1 in the 5'-UTR lowers translation under normal conditions, while deletion of uORF2 elevates it (24). Unlike $M Y C$, we did not identify $A T F 4$ as an EIF3A HLH*-sensitive mRNA (Fig. 1D, table S2-S4). However, we wondered if ATF4 might become sensitive to HLH* EIF3A in the presence of RocA. We transfected CT and HLH* CRISPRi cells with Renilla luciferase reporter mRNAs harboring the ATF4 5'-UTR and saw a modest decrease in luciferase signal in the HLH* cells, normalized to Firefly luciferase reporter mRNA with the $H B B$ 5'-UTR (fig. S5A). Surprisingly, reporter translation increased in the presence of RocA in HLH* but not CT cells, for the mRNAs containing the WT ATF4 5' -UTR (WT) or the ATF4 variant with a mutated start codon in uORF1 ( $\triangle \mathrm{uORF} 1)$. RocA-mediated repression was also relieved in $\mathrm{HLH}^{*}$ cells relative to $\mathrm{CT}$ cells for the $A T F 4$ variant with a mutated start codon in uORF2 ( $\triangle \mathrm{uORF} 2)$ (Fig. 3F). Treatment with thapsigargin (Tg), which induces an ATF4dependent stress response by decreasing active eIF2 levels, had similar effects on translation from these $A T F 4$ reporter mRNAs in the presence of HLH* EIF3A (fig. S5, A and B). By contrast, the combination of RocA and HLH* EIF3A still exhibited a synergistic inhibitory effect on the translation of mRNAs with the MYC 5'-UTR in transfected cells (fig. S5B) as observed in vitro (Fig. 3B). Thus, while HLH* EIF3A does not appear to affect ATF4 uORF translation appreciably (Fig. 3F), it can counterbalance EIF4A1 regulation of certain transcripts, as reflected in the reversal of RocA sensitivity of mRNAs harboring the ATF4 5'-UTR element. 
HLH* EIF3A suppresses MYC-induced proliferation of Burkitt's lymphoma cells

MYC overexpression is responsible for the transformation of Ramos Burkitt's lymphoma cells (25), which prompted us to assess the effect of HLH* EIF3A on proliferation of these MYCaddicted cells. We observed a substantial decrease in MYC and MET protein levels in Ramos Burkitt's lymphoma cell lines expressing the HLH* but not CT EIF3A (Fig. 4A, fig. S6A), more than the mean global decrease in translation in the HLH* Ramos cell lines compared to the CT control cell lines (fig. S6B). To assess the combined effect of HLH* EIF3A and EIF4A1 inhibition, CT and HLH* Ramos cells were cultured overnight in the presence of increasing concentrations of RocA. The HLH* Ramos cells were much more sensitive to RocA compared to CT cells (Fig. 4C). This is consistent with the synergistic sensitization of MYC 5'-UTR observed in in vitro translation extracts (Fig. 3B), and given Ramos cell addiction to MYC overexpression (25). The sensitization effect of $\mathrm{HLH}^{*}$ also occurred in the presence of chemotherapeutic agent doxorubicin (Fig. 4D).

\section{Discussion}

Mammalian eIF3 has been shown to regulate translation initiation of specific mRNAs in a variety of ways: by binding RNA secondary structures in mRNA 5'-UTRs that activate or repress translation, through EIF3D binding to the $\mathrm{m}^{7} \mathrm{G}$ cap, and through $\mathrm{m}^{6} \mathrm{~A}$-dependent interactions with specific mRNAs (3-6). Viral genomic RNAs also target eIF3 to promote translation initiation (26, 27). Structural studies of HCV IRES binding and incorporation into translation preinitiation complexes revealed that the IRES displaces eIF3 from the 40S ribosomal subunit (12). The HCV IRES-driven mode of interaction also requires an HLH RNA binding motif in the EIF3A subunit of eIF3 that is critical for IRES binding and function. Mutation of the putative RNA-binding loop in the HLH motif of EIF3A disrupted eIF3 binding to the IRES and also to the 40S ribosomal subunit (11). Here, using a combination of cell engineering and ribosome profiling, we show that 
mutating the loop in the HLH motif of EIF3A affects the translation of a discrete set of cellular mRNAs. The set of cellular transcripts identified as functionally dependent on the EIF3A HLH motif does not overlap with the eIF3-dependent mRNAs identified previously that require RNA secondary structures (3), rely on EIF3D cap-binding (4), or $\mathrm{m}^{6} \mathrm{~A}$ recognition $(5,6)$, suggesting that the HLH motif in EIF3A contributes to translation of these mRNA using a different mechanism.

In contrast to the HCV IRES, the cellular transcripts sensitive to mutations in the HLH motif of EIF3A are not enriched for putative viral-like RNA secondary structural elements in their 5'-UTRs to which eIF3 could bind (28), and are not enriched for uORFs $(29,30)$. Since the mutation of the HLH motif in EIF3A also disrupts direct binding to the 40S ribosomal subunit (11), translation of the cellular mRNAs identified here may be those most dependent upon the interaction of eIF3 with the 40S subunit. Structural and biochemical evidence has shown eIF3 interacts with the 40S subunit at both the mRNA entry and exit sites within pre-initiation complexes (22). Specifically, the N-terminal domain of EIF3A binds the 40S subunit at the mRNA exit site, while the C-terminal domain projects towards the mRNA entry tunnel. In yeast, mutations in the N-terminal region of eIF3a that weaken mRNA binding to 48S pre-initiation complexes sensitize these to mRNA interactions at the mRNA entry channel, remote from where eIF3a interacts with the 40S subunit. Conversely, mutations in the C-terminus of eIF3a that affect mRNA interactions with the mRNA entry channel also influence mRNA interactions with the mRNA exit site on the opposite side of the 40S subunit. These results reveal a long-distance connection between the two mRNA binding regions in the $48 \mathrm{~S}$ pre-initiation complex important for mRNA recruitment (10). The HLH motif in human EIF3A resides in the N-terminal region that binds at the mRNA exit site and, in yeast, is proposed to generally stabilize recruitment of mRNAs to the $43 \mathrm{~S}$ pre-initiation complex (22). By analogy to the yeast system, the 3 -amino acid 
mutation in the HLH motif in human EIF3A that disrupts eIF3 interactions with the 40S subunit (11) would be predicted to globally decrease favorable interactions of PICs with cellular mRNAs in the mRNA exit channel (Fig. 5A). Global elimination of eIF3-induced mRNA interactions at the mRNA exit site in complexes harboring HLH* EIF3A would then result in decreased translation initiation on transcripts with the least stable interactions in the mRNA entry channel (Fig. 5A), possibly explaining the observed specificity in translation efficiency in HLH* EIF3A expressing cells.

In addition to uncovering cellular mRNAs that may be most sensitive to interactions with the $43 \mathrm{~S}$ pre-initiation complex at the mRNA entry channel, we found that the HLH motif in EIF3A acts as a counterbalance to the action of EIF4A1 during translation initiation. The in vitro translation reactions using $\mathrm{HLH}^{*}$ cell extracts demonstrated that the $\mathrm{HLH}^{*}$ mutations destabilize interactions between EIF3A, the 40S subunit and EIF5B in a transcript-independent manner (Fig. 2), as seen before using a reconstituted system (11). We also found that the HLH mutation destabilizes interactions of initiation complexes with EIF4A1 in a transcript-specific manner, possibly due to weakening eIF3 binding to pre-initiation complexes (Fig. 2) (31). The interaction of the HLH motif in EIF3A and EIF4A1 with the mRNA at the exit and entry points of the initiation complex, respectively, likely affects the dynamics of mRNA scanning to the start codon in a transcript-specific manner, as well as affecting mRNA recruitment $(22,31)$. This transcript specificity is enhanced in the presence of RocA, which locks EIF4A1 onto poly-purine stretches in the 5'-UTR, thereby stalling scanning and mRNA unwinding (18). In the context of an HLHsensitive transcript, RocA exacerbates the translational defect due to HLH* EIF3A (Fig. 3B) by further depleting EIF4A1 from 48S pre-initiation complexes (Fig. 2, E and F, Fig. 3, D and E). By contrast, for transcripts that are not highly reliant on the HLH motif in EIF3A, such as ATF4, RocA repression is alleviated by HLH* EIF3A. Taken together, these results suggest that 
loosening and tightening of mRNA contacts at either end of the mRNA channel in the $48 \mathrm{~S}$ preinitiation complex can lead to combinatorial increases or decreases in translation in a transcriptspecific manner. The mRNA entry and exit channels are spatially separated $(8,10,22)$, implying that HLH* EIF3A loosening of mRNA contacts at the mRNA exit channel of the pre-initiation complex must propagate to the mRNA entry channel to affect the EIF4A1-mRNA interaction, connecting the functions of EIF3A and EIF4A1 during mRNA scanning (Fig. 5).

To test whether the molecular insights gained using HLH* EIF3A could have physiological implications in cancer, we generated HLH* EIF3A expressing Ramos Burkitt's lymphoma cell lines, which are addicted to MYC overexpression for their proliferation (25). We observed that HLH* EIF3A resulted in a dramatic decrease in MYC protein levels and a severe growth defect. The fact that HLH* EIF3A is sufficient to lower MYC levels in these lymphoma cells and increase their sensitivity to chemotherapeutic compounds suggests that eIF3 could serve as a potential target for future cancer therapeutic strategies. Our model for how the HLH motif in EIF3A confers specificity on the translation of specific mRNAs involved in various pathways leading to cell proliferation makes it an intriguing target for treating a wide range of cancers. For example, by combining HLH* EIF3A with RocA treatment, we envision targeting both locations of mRNA engagement with the $48 \mathrm{~S}$ pre-initiation complex, the mRNA entry and exit channels, to achieve selective inhibition of translation. Alternatively, targeting the HLH motif in EIF3A could be used in conjunction with drugs that target completely different pathways, e. g. doxorubicin, a drug thought to target DNA topoisomerases (32) and is commonly used in the treatment of Burkitt's lymphoma. Taken together the EIF3A HLH motif is an attractive new target for drug development to be employed in combination cancer therapy approaches. 


\section{Materials and Methods}

\section{Cell culture}

HEK293T cells were cultured in DMEM (Invitrogen 11995-073) with 10\% FBS (VWR Seradigm 97068-085) and Pen-Strep (10 U/mL) at $37{ }^{\circ} \mathrm{C}$ and 5\% $\mathrm{CO}_{2}$. HBSS was used for washing (Invitrogen 14175-103). Ramos cells were cultured in RPMI 1640 (Thermo 11875-119) with 10\% FBS, 1 mM sodium pyruvate (Thermo 11360-070), 1x NEAA (Thermo 11140-050 100x), and 100 U/mL Pen-Strep (Thermo 15140-122). Lentiviral cell lines were selected with hygromycin (Thermo 10687010, $250 \mu \mathrm{g} / \mathrm{mL}$ ) and puromycin (Mirus MIR5940, $10 \mu \mathrm{g} / \mathrm{mL}$ ). CT or HLH* EIF3A was cloned into nLV103-hygro, and the custom EIF3A shRNA vector was obtained from pLKO.1-puro bacterial stock (see Supplementary Materials and Methods). CRISPRi cell lines were made using catalytically dead Cas9 fused to BFP that was introduced into cells via lentivirus (see Supplementary Methods). Transduced cells were FACS sorted into 96-well plates for clonal amplification, manually screened for BFP expression, and the brightest colonies selected for subsequent introduction of lentiviruses encoding sgRNA in pSLQ1371_BLP1_Ef1A_puro_GFP, a gift from the Jonathan Weissman Lab, and CT or HLH* EIF3A in nLV103-hygro (see Supplementary Methods). Lentiviral vectors were transfected into HEK293T cells to generate viral particles at a ratio of $1 \mu \mathrm{g}: 250 \mathrm{ng}: 250 \mathrm{ng}$ lentivirus carrying the gene of interest, $\mathrm{dR} 8.91$ packaging vector, and pMD2.G envelope vector, respectively, per each well in a 6-well format. The viral supernatant was harvested at 48 hours and transduced into relevant cell lines using 20 $\mu \mathrm{g} / \mathrm{mL}$ polybrene. DNA and RNA transfections were performed using Opti-MEM Reduced Serum Media (Invitrogen 31985-088) and TransIT-2020 (MIR5404) and TransIT-mRNA (MIR2225) reagents according to the manufacturer's protocol (Mirus). 


\section{Ribosome Profiling}

Ribosome profiling libraries were prepared from three biological replicates per cell line according to previously described methods (33). RNAseq libraries were prepared from the same samples using TruSeq RNA Library Prep kit according to the manufacturer's instructions (Illumina Part \# 15026495). Sequencing data were analyzed using Bowtie v1.0.0 (34) to remove rRNA reads, TopHat v2.0.14 $(35,36)$ to align reads to the human GRCh38 genome, Cufflinks v2.2.1 and Cuffdiff v2.2.1 (35) to extract and merge raw read counts of the biological replicates, and $\mathrm{R}$ v3.2.2 package Babel v0.2-6: Ribosome Profiling Analysis to calculate FPKM, $p$-values and FDR (R Core Team (2015). R: A language and environment for statistical computing. R Foundation for Statistical Computing, Vienna, Austria https://www.R-project.org/). Translational efficiency (TE) was calculated as ribosome profiling FPKM / RNAseq FPKM and fold change was calculated as HLH* TE / CT TE. See tables S2-S4 for data (raw non-zero, Babel output). Bowtie2 (37) was used to generate indices from EIF3A CT and HLH* sequences for alignment with CT and HLH* RNAseq data (see Supplementary Methods). All data has been deposited in Gene Expression Ombinus (accession number

\section{5'-UTR $u O R F$ and secondary structure computational analysis}

We used the databases uORFdb (29) (uORFdb - a comprehensive literature database on eukaryotic uORF biology, http://www.compgen.uni-muenster.de/tools/uorfdb/) and TISdb (30)

(Translation Initiation Site Database, http://tisdb.human.cornell.edu/) to analyze transcripts

affected by HLH* EIF3A for uORF presence. We used RNAstructure v6.0.1 Secondary Structure Web Server (28) (https://rna.urmc.rochester.edu/RNAstructureWeb/) to predict secondary structures of transcripts affected by HLH* EIF3A. 


\section{Western Blotting}

The following antibodies were used for Western blot analysis using the manufacturers' suggested dilutions: anti-beta-Actin (Abcam ab8227), anti-ATF4 (Abcam ab184909), anti-DEPTOR (Sigma SAB4200214), anti-eIF1A (Abcam ab177939), anti-eIF2 $\alpha$ (Bethyl A300-721A-M), anti-eIF3A

(Sigma SAB1402997-100UG), anti-eIF4A1 (Abcam ab31217), anti-eIF5B (Bethyl A301-745AM), anti-HSP90 (Abcam ab13492), anti-MET (Abcam ab51067), anti-MYC (Abcam ab32072), anti-PTP4A3 (Abcam ab50276 recognizes PRL-3), anti-RPS19 (Bethyl A304-002A), anti-Mouse IgG-HRP (A00160), anti-Rabbit IgG-HRP (NA934V). Protein levels in Western blots were quantified using ImageJ (38).

\section{In vitro transcription}

RNAs were transcribed from $1 \mu \mathrm{g}$ of PCR-amplified templates using T7 RNA polymerase in 1x transcription buffer $(30 \mathrm{mM}$ Tris- $\mathrm{Cl} \mathrm{pH} 8,5 \mathrm{mM} \mathrm{MgCl} 2,0.01 \%$ Triton X-100, $2 \mathrm{mM}$ spermidine, $20 \mathrm{mM}$ NTPs, $10 \mathrm{mM}$ DTT) for 5 hrs at $37^{\circ} \mathrm{C}$. Reactions were treated with RQ1 DNAse (Promega M6101) for $20 \mathrm{~min}$ at $37^{\circ} \mathrm{C}$, precipitated using 2x volume $7.5 \mathrm{M} \mathrm{LiCl} / 50 \mathrm{mM}$ EDTA at $-20{ }^{\circ} \mathrm{C}$ for $30 \mathrm{~min}$, washed $2 \mathrm{x}$ in $70 \% \mathrm{EtOH}$, and resuspended in RNase free water. RNAs were capped using the Vaccinia capping system (NEB M2080S) according to manufacturer's protocol, in the presence of $100 \mathrm{U}$ murine RNase inhibitor (M0314S), extracted with an equal volume of phenol:chloroform $\mathrm{pH} 6$, precipitated at $-20{ }^{\circ} \mathrm{C}$ overnight in $5 \mathrm{x}$ volume $2 \% \mathrm{LiClO}_{4}$ in acetone, washed $2 \mathrm{x}$ in $70 \% \mathrm{EtOH}$ and resuspended in RNase free water. RNAs that were amplified without a poly-A tail were poly-adenylated using poly-A polymerase (NEB M0276) according to the manufacturer's protocol.

\section{In vitro translation}


Cell extracts were prepared from CRISPRi-engineered HEK293T cell lines at $\sim 80 \%$ confluency. Cells were washed and scraped in cold PBS, spun down for $5 \mathrm{~min}$ at $1000 \mathrm{~g}$ at $4{ }^{\circ} \mathrm{C}$, and resuspended in an equal volume of hypotonic lysis buffer (10 mM HEPES 7.6, 0.5 mM MgOAc, 5 mM DTT, Halt protease/phosphatase inhibitor cocktail (Thermo 78440)) for 45 min. Extracts were homogenized $\sim 20$ times through a $27 \mathrm{G}$ needle, spun down for $1 \mathrm{~min}$ at $14,000 \mathrm{~g}$ at $4{ }^{\circ} \mathrm{C}$, and the supernatant removed, avoiding the lipids on the top and interface on the bottom. In vitro translation reactions with luciferase reporter mRNAs were carried out with $0.5 \mathrm{x}$ extract, energy mix (final $0.84 \mathrm{mM}$ ATP, $0.21 \mathrm{mM} \mathrm{GTP,} 21 \mathrm{mM}$ creatine phosphate (Roche 10621722001), 45 U/mL creatine phosphokinase (Roche 10127566001), 10 mM HEPES pH 7.5, 2 mM DTT, 2.5 mM MgOAc, 50 mM KOAc, $8 \mu \mathrm{M}$ amino acids (Promega PRL4461), $255 \mu \mathrm{M}$ spermidine, 1U/mL murine RNase inhibitor (NEB M0314)), and 400 ng total RNA. Rocaglamide A (RocA, a gift from the Nicholas Ingolia Lab) was added to a final concentration of $0.1 \mu \mathrm{M}$ where indicated. Reactions were incubated for $1 \mathrm{hr}$ at $30^{\circ} \mathrm{C}$ and luciferase signal was measured using Dual-Glo Luciferase Assay System (Promega E2920). In vitro translation extracts for sucrose gradient fractionation were first treated with micrococcal nuclease (NEB M0247S) and $0.8 \mathrm{mM} \mathrm{CaCl}_{2}$ for $10 \mathrm{~min}$ at $25^{\circ} \mathrm{C}$. Treatment was stopped with $3.2 \mathrm{mM}$ EGTA. Treated extracts were then mixed in a 2:1:1 ratio with the energy mix and $1 \mu \mathrm{g}$ full-length $M Y C$ or $G A P D H$ mRNA in water and incubated for $20 \mathrm{~min}$ at $30{ }^{\circ} \mathrm{C}$ prior to loading on gradients. Cycloheximide $(100 \mu \mathrm{g} / \mathrm{mL}$; Sigma 01810) or GMP-PNP (Sigma G0635) were added to the energy mix prior to the translation reaction. GMP-PNP was added at $0.21 \mathrm{mM}$ instead of GTP.

\section{Sucrose gradient fractionation}

In vitro translation reactions were sedimented on 10-25\% sucrose gradients (containing $20 \mathrm{mM}$ HEPES pH 7.5, 150 mM KOAc, 2.5 mM MgOAc, 1 mM DTT, 0.2 mM spermidine, $100 \mu \mathrm{g} / \mathrm{mL}$ cycloheximide if reaction contained cycloheximide) for $3.5 \mathrm{hrs}$ at $240,000 \mathrm{~g}$ at $4{ }^{\circ} \mathrm{C}$ using a SW41 
rotor (Beckman Coulter). Gradients were fractionated using Teledyne Isco Tris Peristaltic Pump and fractions were collected and pooled according to the UV trace. Fractions were concentrated using Amicon $30 \mathrm{kDa}$ spin columns (UFC503096) according to the manufacturer's instructions. For Northern blot analysis, fractions were treated with 1\% SDS and 1\% Proteinase K solution (20 $\mathrm{mg} / \mathrm{mL}$ Proteinase K (Thermo 26160), 0.2 M Tris-HCl pH 7.5, 0.2 M NaCl, $1.5 \mathrm{mM} \mathrm{MgCl}_{2}$ ) at 42 ${ }^{\circ} \mathrm{C}$ for 30 min. RNA was extracted using an equal volume of phenol:chloroform $\mathrm{pH} 6$, precipitated at $-20^{\circ} \mathrm{C}$ overnight in $2 \mathrm{x}$ volume $100 \% \mathrm{EtOH}, 2.7 \mathrm{M} \mathrm{NaOAc}$, and $10 \mu \mathrm{g} / \mathrm{mL}$ GlycoBlue Coprecipitant (Thermo AM9515), washed 2x in 70\% EtOH and resuspended in RNase free water.

\section{Northern Blotting}

Total RNA isolated from the sucrose gradient fractions was resolved using a $10 \%$ polyacrylamide gel in $0.5 x$ TBE buffer buffer (1x TBE buffer contains $89 \mathrm{mM}$ Tris, $89 \mathrm{mM}$ boric acid, and $2 \mathrm{mM}$ EDTA) and electroblotted onto a nylon $(\mathrm{N}+)$ membrane (GE Healthcare RPN203B) at $20 \mathrm{~V}$ for $90 \mathrm{~min}$ at $4{ }^{\circ} \mathrm{C}$ in $0.5 \mathrm{x}$ TBE buffer. The membrane was crosslinked and pre-hybridized in UltraHyb Hybridization Solution (Thermo AM8670) at $42{ }^{\circ} \mathrm{C}$ for 1 hour, then incubated overnight with 50 pmol Met-tRNA ${ }_{i}$ specific probe (5'-TGGTAGCAGAGGATGGTTTCGAT-3'). The probe was labeled on the $5^{\prime}$ end with $[\gamma-32 \mathrm{P}]$ ATP (Perkin Elmer) using T4 polynucleotide kinase (NEB M0201) according to the manufacturer's protocol. Membranes were washed twice by $20 \mathrm{~mL} 6 \mathrm{x} \mathrm{SSC}$ for $5 \mathrm{~min}$ at $42{ }^{\circ} \mathrm{C}$ and twice by $20 \mathrm{~mL} 2 \mathrm{x} \mathrm{SSC}$ and twice by $20 \mathrm{~mL} 1 \mathrm{x}$ SSC (20x SSC contains $0.3 \mathrm{M}$ sodium citrate in $3 \mathrm{M} \mathrm{NaCl}$ ). Membranes were then wrapped in saran wrap, exposed to a phosphor screen overnight, and visualized by phoshor-imaging.

\section{Cell viability assays}


Ramos cells were seeded at $1 \times 10^{6}$ cells $/ \mathrm{mL}$ into 96 -well plates in the presence or absence of drug (RocA, gift from Nicholas Ingolia Lab, $0-0.1 \mu \mathrm{M}$; Doxorubicin (Fisher BP25161), $0-4$ $\mu \mathrm{M})$, cultured for 24 hours, and cell viability was assessed using CellTiter-Glo assay according to the manufacturer's protocol (Promega G7570).

\section{Metabolic labeling}

Cells were seeded at $1 \times 10^{6}$ cells $/ \mathrm{mL}$ into 6 -well plates and allowed to adhere and grow overnight. Media was changed to DMEM -Met/Cys (Thermo 21013024) for $30 \mathrm{~min}$, then each well was incubated for $30 \mathrm{~min}$ at $37^{\circ} \mathrm{C}$ with $5 \mu \mathrm{l} /$ well EXPRE35S35S Protein Labeling Mix (PerkinElmer NEG072002MC), after which cells were lysed in RIPA lysis buffer (50 mM Tris$\mathrm{HCl} \mathrm{pH}$ 7.5, $150 \mathrm{mM} \mathrm{NaCl}, 0.25 \%$ deoxycholic acid, $1 \% \mathrm{NP}-40,1 \mathrm{mM}$ EDTA). Lysates were boiled in SDS loading buffer, resolved on SDS-PAGE gels, and stained with Coommassie to visualize total protein. Gels were dried at $80^{\circ} \mathrm{C}$ for 1 hour on a gel drier, exposed to a phosphor screen overnight, and ${ }^{35} \mathrm{~S}$ incorporation was visualized by phoshor-imaging.

Plasmids and gene sequences are included in Supplementary Materials and Methods. 


\section{References}

1. D. Ruggero, Translational control in cancer etiology. Cold Spring Harb Perspect Biol. 5, a012336-a012336 (2013).

2. A. G. Hinnebusch, J. R. Lorsch, The mechanism of eukaryotic translation initiation: new insights and challenges. Cold Spring Harb Perspect Biol. 4, a011544-a011544 (2012).

3. A. S. Y. Lee, P. J. Kranzusch, J. H. D. Cate, eIF3 targets cell-proliferation messenger RNAs for translational activation or repression. Nature. 522, 111-114 (2015).

4. A. S. Y. Lee, P. J. Kranzusch, J. A. Doudna, J. H. D. Cate, eIF3d is an mRNA cap-binding protein that is required for specialized translation initiation. Nature. 536, 96-99 (2016).

5. K. D. Meyer et al., 5' UTR m6A Promotes Cap-Independent Translation. Cell. 163, 9991010 (2015).

6. X. Wang et al., N6-methyladenosine Modulates Messenger RNA Translation Efficiency. Cell. 161, 1388-1399 (2015).

7. A. des Georges et al., Structure of mammalian eIF3 in the context of the 43S preinitiation complex. Nature. 525, 491-495 (2015).

8. Y. Hashem et al., Structure of the Mammalian Ribosomal 43S Preinitiation Complex Bound to the Scanning Factor DHX29. Cell. 153, 1108-1119 (2013).

9. M. Sokabe, C. S. Fraser, J. W. B. Hershey, The human translation initiation multi-factor complex promotes methionyl-tRNA i binding to the 40S ribosomal subunit. Nucleic Acids Research. 40, 905-913 (2011).

10. B. Eliseev et al., Structure of a human cap-dependent $48 \mathrm{~S}$ translation pre-initiation complex. Nucleic Acids Research. 46, 2678-2689 (2018).

11. C. Sun et al., Two RNA-binding motifs in eIF3 direct HCV IRES-dependent translation. Nucleic Acids Research. 41, 7512-7521 (2013).

12. Y. Hashem et al., Hepatitis-C-virus-like internal ribosome entry sites displace eIF3 to gain access to the 40S subunit. Nature. 503, 539-543 (2013).

13. J. P. Erzberger et al., Molecular Architecture of the 40SSeIF1SeIF3 Translation Initiation Complex. Cell. 158, 1123-1135 (2014).

14. H. Chen, H. Liu, G. Qing, Targeting oncogenic Myc as a strategy for cancer treatment. Signal Transduction and Targeted Therapy, 1-7 (2018).

15. E. Gherardi, W. Birchmeier, C. Birchmeier, G. V. Woude, Targeting MET in cancer: rationale and progress. Nat. Rev. Cancer. 12, $89 \mathrm{EP}-$.

16. Z. Ye et al., PRL-3 activates mTORC1 in Cancer Progression. Nature Publishing Group, $1-15$ (2015). 
17. S. E. Dmitriev, A. V. Pisarev, M. P. Rubtsova, Y. E. Dunaevsky, I. N. Shatsky, Conversion of $48 \mathrm{~S}$ translation preinitiation complexes into $80 \mathrm{~S}$ initiation complexes as revealed by toeprinting. FEBS Lett. 533, 99-104 (2003).

18. S. Iwasaki, S. N. Floor, N. T. Ingolia, Rocaglates convert DEAD-box protein eIF4A into a sequence-selective translational repressor. Nature. 534, 558-561 (2016).

19. S. Gunišová, P. Beznosková, M. P. Mohammad, V. Vlčková, L. S. Valášek, In-depth analysis of cis-determinants that either promote or inhibit reinitiation on GCN4mRNA after translation of its four short uORFs. RNA. 22, 542-558 (2016).

20. V. Munzarová et al., Translation Reinitiation Relies on the Interaction between eIF3a/TIF32 and Progressively Folded cis-Acting mRNA Elements Preceding Short uORFs. PLoS Genet. 7, e1002137-16 (2011).

21. B. Szamecz et al., eIF3a cooperates with sequences 5' of uORF1 to promote resumption of scanning by post-termination ribosomes for reinitiation on GCN4 mRNA. Genes \& Development. 22, 2414-2425 (2008).

22. C. E. Aitken et al., Eukaryotic translation initiation factor 3 plays distinct roles at the mRNA entry and exit channels of the ribosomal preinitiation complex. Elife. 5, 111 (2016).

23. R. Watanabe et al., The eukaryotic initiation factor (eIF) 4G HEAT domain promotes translation re-initiation in yeast both dependent on and independent of eIF4A mRNA helicase. J. Biol. Chem. 285, 21922-21933 (2010).

24. K. M. Vattem, R. C. Wek, Reinitiation involving upstream ORFs regulates ATF4 mRNA translation in mammalian cells. Proc Natl Acad Sci USA. 101, 11269-11274 (2004).

25. M.-E. Habel, D. Jung, c-Myc over-expression in Ramos Burkitt's lymphoma cell line predisposes to iron homeostasis disruption in vitro. Biochem. Biophys. Res. Commun. 341, 1309-1316 (2006).

26. D. V. Sizova, V. G. Kolupaeva, T. V. Pestova, I. N. Shatsky, C. U. Hellen, Specific interaction of eukaryotic translation initiation factor 3 with the $5^{\prime}$ nontranslated regions of hepatitis C virus and classical swine fever virus RNAs. J. Virol. 72, 4775-4782 (1998).

27. H. Ji, C. S. Fraser, Y. Yu, J. Leary, J. A. Doudna, Coordinated assembly of human translation initiation complexes by the hepatitis $\mathrm{C}$ virus internal ribosome entry site RNA. Proc Natl Acad Sci USA. 101, 16990-16995 (2004).

28. D. H. Mathews, Using the RNAstructure Software Package to Predict Conserved RNA Structures. Curr Protoc Bioinformatics. 46, 12.4.1-22 (2014).

29. K. Wethmar, A. Barbosa-Silva, M. A. Andrade-Navarro, A. Leutz, uORFdb--a comprehensive literature database on eukaryotic uORF biology. Nucleic Acids Research. 42, D60-7 (2014).

30. S. Lee et al., Global mapping of translation initiation sites in mammalian cells at singlenucleotide resolution. Proc. Natl. Acad. Sci. U.S.A. 109, E2424-32 (2012). 
31. P. Yourik et al., Yeast eIF4A enhances recruitment of mRNAs regardless of their structural complexity. Elife. 6, 115 (2017).

32. J. L. Nitiss, Targeting DNA topoisomerase II in cancer chemotherapy. Nat. Rev. Cancer. 9, 338-350 (2009).

33. N. T. Ingolia, G. A. Brar, S. Rouskin, A. M. McGeachy, J. S. Weissman, Genome-Wide Annotation and Quantitation of Translation by Ribosome Profiling (John Wiley \& Sons, Inc., Hoboken, NJ, USA, 2001), vol. 11.

34. B. Langmead, C. Trapnell, M. Pop, S. L. Salzberg, Ultrafast and memory-efficient alignment of short DNA sequences to the human genome. Genome Biol. 10, R25 (2009).

35. C. Trapnell et al., Differential gene and transcript expression analysis of RNA-seq experiments with TopHat and Cufflinks. Nat Protoc. 7, 562-578 (2012).

36. D. Kim et al., TopHat2: accurate alignment of transcriptomes in the presence of insertions, deletions and gene fusions. Genome Biol. 14, R36 (2013).

37. B. Langmead, S. L. Salzberg, Fast gapped-read alignment with Bowtie 2. Nat. Methods. 9, 357-359 (2012).

38. C. A. Schneider, W. S. Rasband, K. W. Eliceiri, NIH Image to ImageJ: 25 years of image analysis. Nat. Methods. 9, 671-675 (2012).

39. M. D. Smith et al., Assembly of eIF3 Mediated by Mutually Dependent Subunit Insertion. Structure. 24, 886-896 (2016).

40. M. A. Horlbeck et al., Compact and highly active next-generation libraries for CRISPRmediated gene repression and activation. Elife. 5, 914 (2016). 


\section{Acknowledgements}

The authors thank Amy S.Y. Lee, Nathanael Lintner, M. Duane Smith, Audrone Lapinaite and Michael Rape for helpful discussions. We also thank Jonathan Weissman and Jacob Corn for CRISPRi-related plasmids, and Nicholas Ingolia and Shintaro Iwasaki for RocA and helpful

feedback. This work was supported by National Institutes of Health Grants R01-GM065050 and P50 GM102706 (to J.H.D.C.). This work used the Vincent J. Coates Genomics Sequencing Laboratory at UC Berkeley, supported by the NIH Instrumentation Grant S10 OD018174. 


\section{Figure Legends}

Fig. 1. EIF3A HLH motif regulates translation of cancer-associated RNAs. A. Schematic of eIF3 binding to the 40S ribosomal subunit in canonical and viral IRES-mediated initiation complexes based on cryo-EM reconstructions $(8,12)$. B. In vitro translation of IRES-Renilla mRNA in CT and HLH* extracts. Error bars indicate the standard deviation of biological triplicates. C. Representative alignment of RNAseq CT and HLH reads to wild type (wt) or HLH* (mut) eIF3A sequences. D. Translational efficiency scatter plot of statistically significant transcripts ( $p$-value $<0.01)$. Upregulated transcripts highlighted in blue ( $>3 \mathrm{x}$ increase), downregulated in pink ( $>3 \mathrm{x}$ decrease). E. Functional classification of regulated transcripts based on literature analysis. F. Representative Western blot validation of top cancer associated hits in shRNA 293 T cell lines. Levels of protein normalized to ACTB or HSP90 control given below gels. G. Representative Western blot in CRISPRi 293 T cell lines. Levels of protein normalized to ACTB or HSP90 control given below gels.

\section{Fig. 2. General and transcript-specific defects in initiation factor recruitment}

A, B. Representative sucrose gradient profile of in vitro translation reactions stalled with cycloheximide or GMPPNP and programmed with full length mRNA. Fractions were tracked by absorbance at $254 \mathrm{~nm}$ as shown, with the top of the gradient on the left. C, D. Western blot analysis of initiation factor distribution in translation reactions stalled with cycloheximide. E, F. Western blot analysis of initiation factor distribution in translation reactions stalled with GMPPNP. Red boxes indicate fractions with decreased levels of initiation factors of interest. 
Fig. 3. EIF3A HLH motif interacts with specific mRNA 5'-UTR elements. A. In vitro translation of HCV IRES and MYC 5'-UTR Renilla mRNAs in CT and HLH* extracts. The schematic on the right shows the features and design of the mRNAs used. Globin $H B B$ 5'-UTR Firefly mRNA was used as a control. Statistical significance measured by student's t-test, $* * * P<$ 0.0001. Error bars represent the standard deviation between technical triplicates.

B. In vitro translation of HCV IRES and MYC 5'-UTR luciferase mRNAs in the presence of EIF4A1 inhibitor RocA. Statistical significance measured by student's t-test, ${ }^{*} P<0.01 ; * * P<$ $0.001 ; * * * P<0.0001$. Error bars represent the standard deviation between technical triplicates. C-E. In vitro translation reactions programmed with full length $G A P D H$ and $M Y C$ mRNAs inhibited with both GMPPNP and RocA and fractionated on 10-25\% sucrose gradients. Western blotting of the sucrose gradient fractions, with red boxes indicating fractions of interest for EIF4A1 levels. Yellow asterisk indicates background signal in gel, which does not interfere with initiation factor distribution analysis. F. EIF3A HLH* motif effects on RocA-mediated repression. The schematic on the right represents the ATF4 uORF variant 5'-UTRs (WT, $\triangle \mathrm{uORF} 1, \Delta \mathrm{uORF} 2)$ fused to the Renilla luciferase ORF for transfection into CRISPRi CT and HLH* $^{*}$ cells. Live cell transfection was performed instead of in vitro translation in order to observe the effect of RocA stress. Relative Luciferase Units (RLU) percentage was normalized to internal globin $H B B$ 5'-UTR Firefly mRNA control signal. Statistical significance was measured by student's t-test, $* P<0.01, * * P<0.001, * * * P<0.0001$. Error bars represent the standard deviation between technical triplicates.

Fig. 4. HLH* EIF3A effects in Ramos Burkitt's lymphoma cells. A. Western blot analysis of HLH* sensitive proteins in Ramos shRNA lentiviral cell lines. Levels of protein normalized to ACTB control given below gels. Asterisk notes that no detectable PRL3 was found in Ramos cells. B. CT and HLH* Ramos cells cultured in the presence of increasing concentrations of 
RocA. Error bars represent standard deviation of three biological replicates. C. Doxorubicin treatment of Ramos CT (R-CT) and HLH* (R-HLH) cell lines.

Fig. 5. Model of EIF4A1 and EIF3A dynamic interactions with mRNA entry and exit sites.

A. Schematic of EIF4A1 and eIF3 interacting with the mRNA at the entry and exit mRNA sites of the 40 S ribosomal subunit, respectively. B. Schematic representing the displacement of EIF3 HLH* $^{*}$ from the 40S subunit that leads to general defects in initiation factor recruitment and mRNA translation. C. Schematic representing the displacement of EIF3 HLH* from the 40S subunit in the presence of select transcripts, such as $M Y C$, and the concomitant displacement of EIF4A1 at the entry site. 


\section{Figure 1}
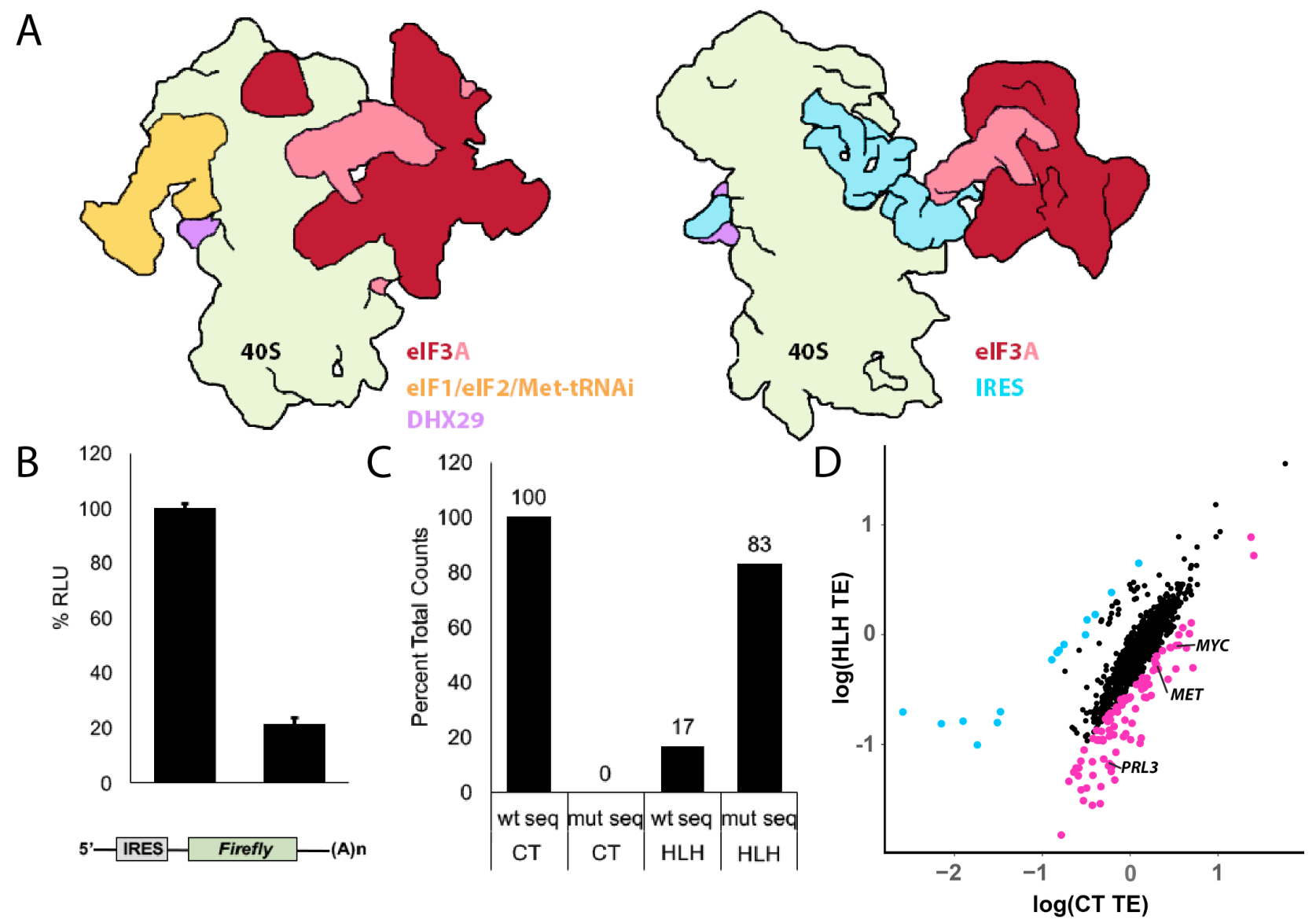

$\mathrm{E}$

F

Translationally Downregulated Genes

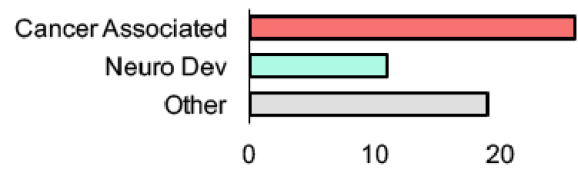

Translationally Upregulated Genes

30

G
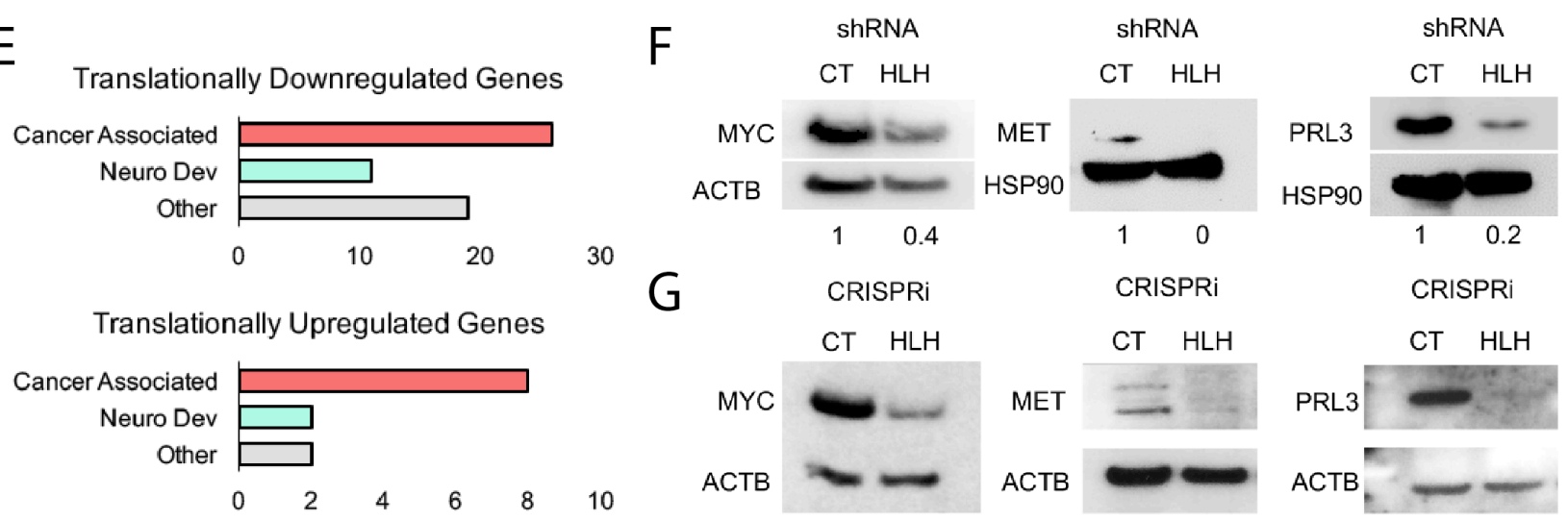

10
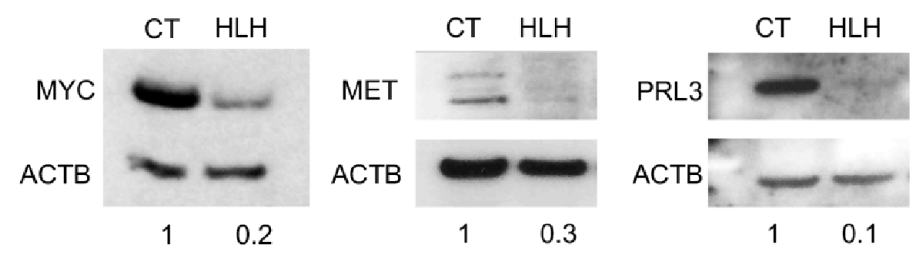


\section{Figure 2}

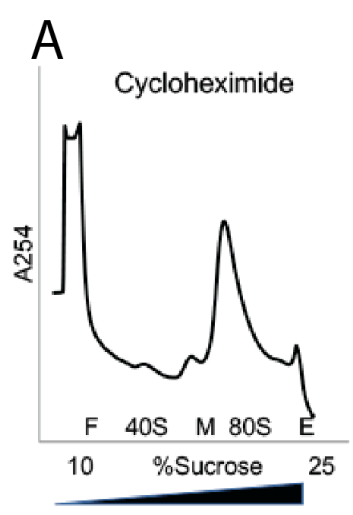

B

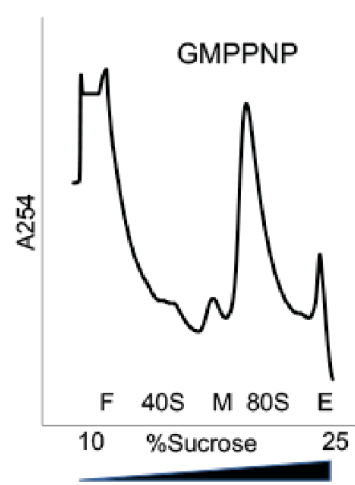

C

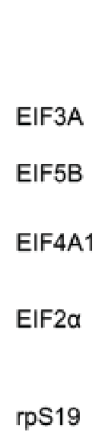

$E$

D

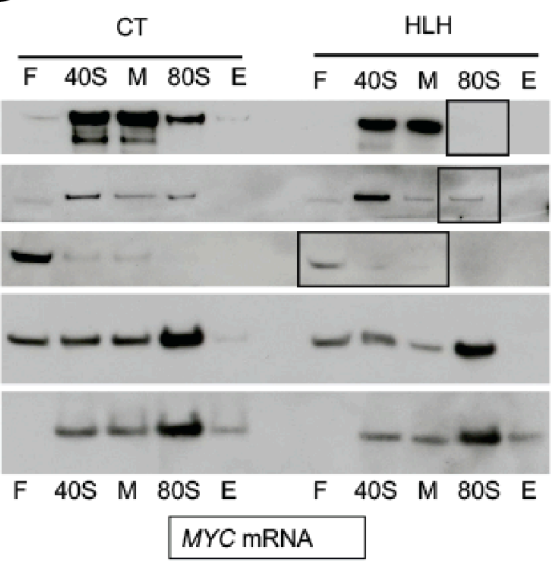

$\mathrm{F}$

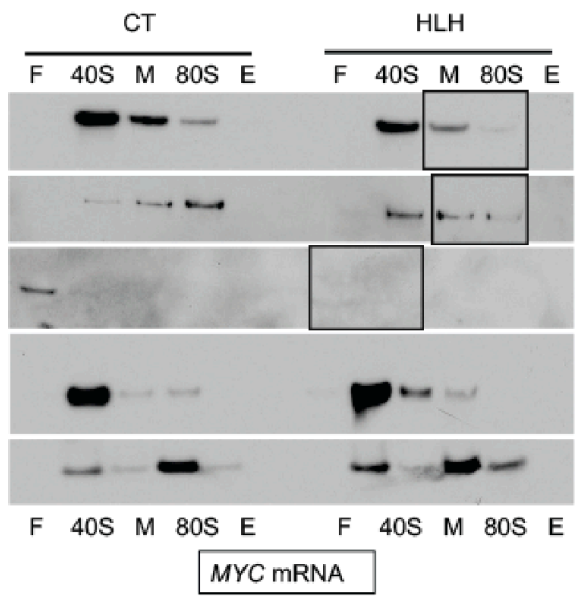




\section{Figure 3}

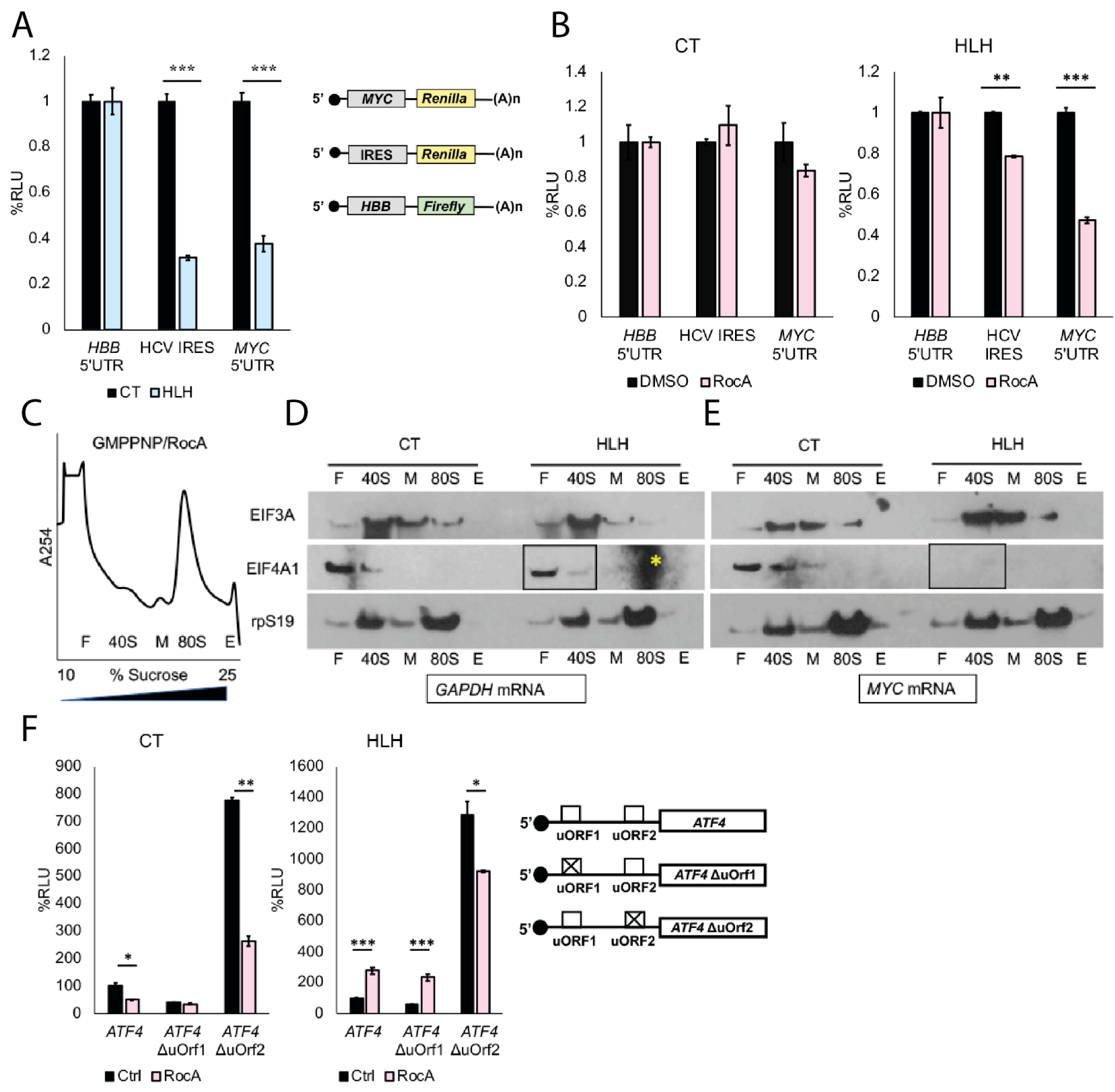




\section{Figure 4}

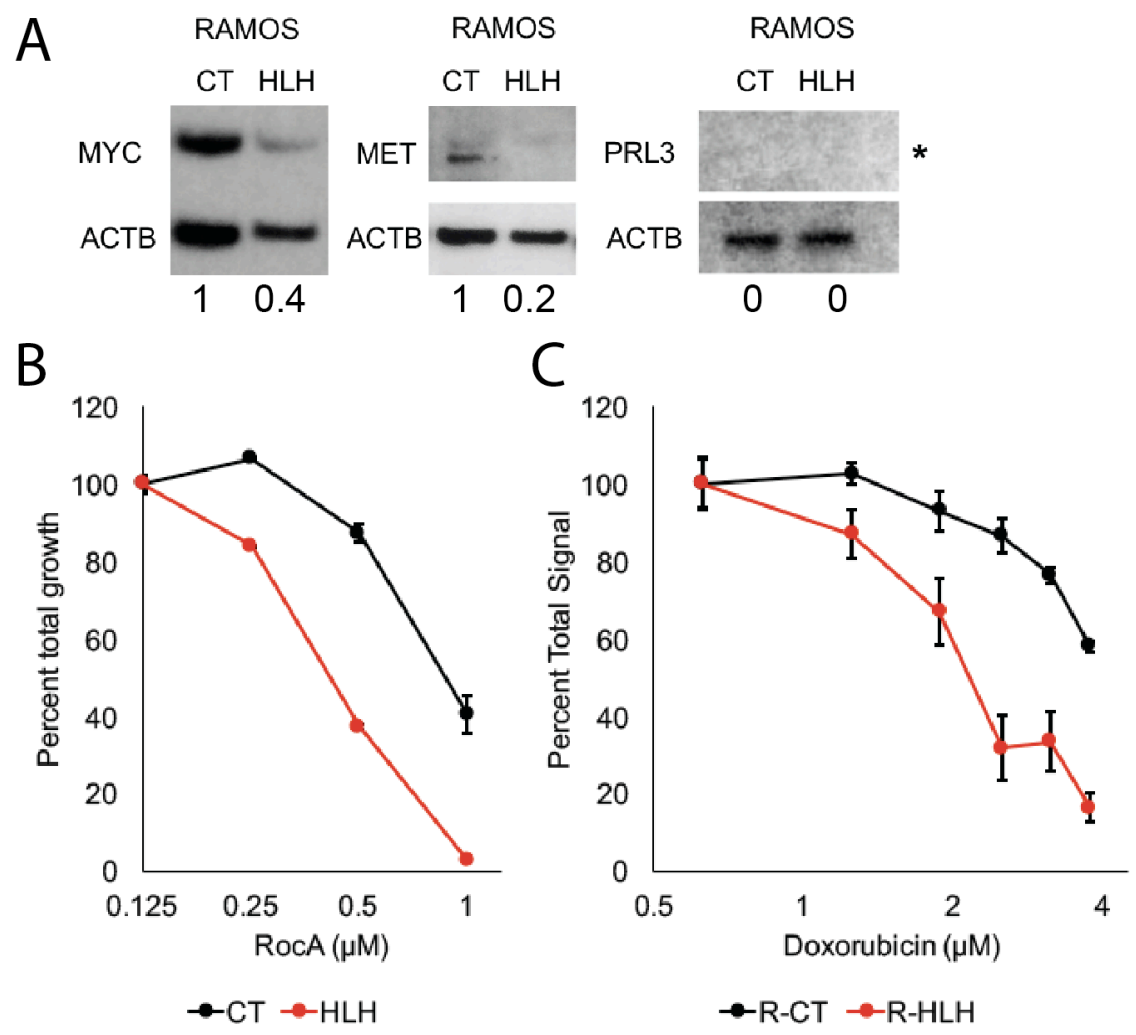




\section{Figure 5}

A

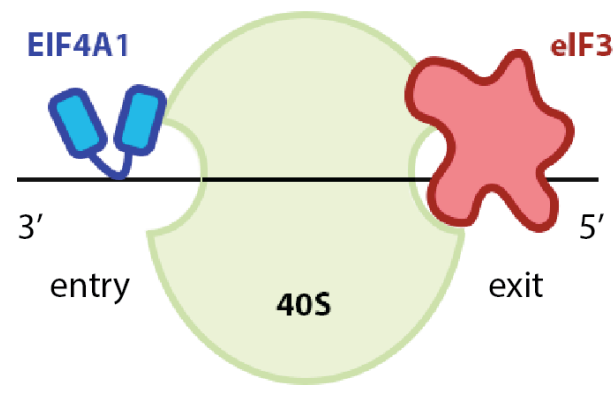

B

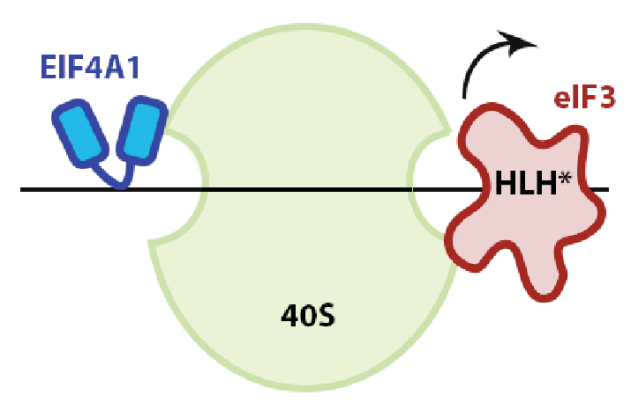

C

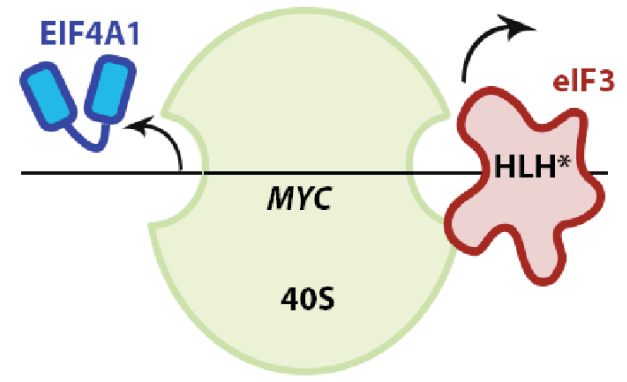


Table 1. Cell lines used in this study and the method of engineering.

\begin{tabular}{|l|l|l|l|}
\hline Cell Line & Endogenous KD & Exogenous & Selection \\
\hline CT & shRNA & eIF3A, shRNA & hygromycin, puromycin \\
\hline HLH & shRNA & eIF3A HLH*, shRNA & hygromycin, puromycin \\
\hline CT2SG3 & dCas9/sgRNA & dCas9, sgRNA, eIF3A & BFP, hygromycin, puromycin \\
\hline HLH6SG3 & dCas9/sgRNA & dCas9, sgRNA, eIF3A HLH* & BFP, hygromycin, puromycin \\
\hline R-CT & shRNA & eIF3A, shRNA & hygromycin, puromycin \\
\hline R-HLH & shRNA & eIF3A HLH*, shRNA & hygromycin, puromycin \\
\hline
\end{tabular}




\section{Supplementary Materials}

\section{Cloning}

EIF3A was PCR-amplified from HEK293T cDNA and cloned into vector nLv-103 (hygromycin) (39) using PCR-based restriction free cloning. HLH mutations and shRNA target site mutations were introduced by PCR-based site-directed mutagenesis. Custom EIF3A shRNA lentiviral vector was obtained from Sigma-Aldrich MISSION TRC shRNA pLKO.1-puro bacterial stock (Sigma SHCLNG). pMD2.G (Addgene \#12259) envelope protein vector and pCMV-dR8.91 (Addgene \# 2221) packaging vector were used to make viral particles for transduction. The same vectors were later used to package CRISPRi lentiviral plasmids. HCV IRES, MYC 5'-UTR, and ATF4 5'-UTR elements were cloned into pcDNA4-Rluc using HinDIII and EcoRI restriction sites; the HBB 5'UTR was cloned into pcDNA4-Fluc, where the Renilla luciferase ORF sequence was replaced by the Firefly luciferase ORF. pCMV-SPORT6 containing MGC Human MYC cDNA (CloneId:2985844) glycerol stock was obtained from Dharmacon (MHS6278). Full-length MYC and GAPDH were amplified from cDNA and cloned into pCMV-SPORT6 using SalI and NotI restriction sites. pHR-EF1a-dCas9-HA-BFP-KRAB-NLS, a gift from the Jacob Corn Lab (Addgene plasmid \#102244), was used to introduce catalytically dead Cas9 into HEK293T cell lines. EIF3A sgRNA sequences were obtained from the human CRISPRi library v2 (40) and cloned into in pSLQ1371_BLP1_Ef1A_puro_GFP, a gift from the Jonathan Weissman Lab, using BstX1 and Blp1 restriction sites. The EIF3A sgRNA top and bottom oligo were ordered from IDT (for final selected sgRNA: (top) 5'- TTGGCAGCCGGCCAGAGACGGAAGTTTAAGAGC -3'; (bottom) 5' - TTAGCTCTTAAACTTCCGTCTCTGGCCGGCTGCCAACAAG -3') and annealed by incubating at $95^{\circ} \mathrm{C}$ for $5 \mathrm{~min}$ in annealing buffer (100 mM Potassium acetate, $30 \mathrm{mM}$ HEPES$\mathrm{KOH} \mathrm{pH}$ 7.4, $2 \mathrm{mM} \mathrm{Mg}$ acetate) and slowly cooling to room temperature. $5 \mathrm{nM}$ annealed oligo 
were ligated to $10 \mathrm{ng}$ digested vector backbone using T4 ligase (Thermo A13726) according to the manufacturer's protocol.

EIF3A mRNA (site of HLH mutations indicated in red)

ATGCCGGCCTATTTTCAGAGGCCGGAAAATGCCCTCAAACGCGCCAACGAATTTCTT GAGAACATAATTGGTTCGTGTATCTTTTGCCATGTTCTTTATGATGTTATGAAAAGTA AAAAACATAGAACATGGCAAAAGATACACGAACCAATTATGTTGAAATACTTGGAA CTTTGCGTGGATCTTCGCAAGAGCCACTTGGCAAAGGAGGGGTTATACCAGTATAAG AACATTTGTCAACAGGTGAACATAAAATCTCTGGAGGATGTTGTTAGGGCATATTTG AAAATGGCAGAGGAAAAAACTGAAGCTGCTAAAGAAGAATCTCAGCAGATGGTCTT AGATATAGAGGATCTAGATAATATTCAAACTCCTGAGAGTGTTCTCCTAAGTGCTGT AAGTGGTGAAGACACTCAGGATCGTACTGACAGATTACTTTTAACTCCATGGGTTAA ATTCCTGTGGGAGTCTTACAGGCAGTGTTTGGACCTTCTTAGAAACAATTCTAGAGTA GAGCGCCTGTACCATGATATTGCCCAGCAAGCTTTCAAATTCTGCCTCCAATACACGC GTAAGGCTGAATTCCGTAAACTGTGTGACAATTTGAGAATGCACTTATCGCAGATTC AGCGCCACCATAACCAAAGTACGGCAATCAATCTTAATAATCCAGAGAGCCAGTCCA TGCATTTGGAAACCAGACTTGTTCAGCTGGACAGTGCTATCAGCATGGAATTGTGGC AGGAAGCATTCAAAGCTGTGGAAGATATTCACGGGCTATTCTCCTTGTCTAAAAAAC CACCTAAACCTCAGTTGATGGCAAATTACTATAACAAAGTCTCAACTGTGTTTTGGAA ATCTGGAAATGCTCTTTTTCATGCATCTACACTCCATCGTCTTTACCATCTCTCTAGAG AAATGAGAAAGAATCTCACACAAGATGAGATGCAAAGAATGTCTACTAGAGTCCTTT TAGCCACTCTTTCCATCCCTATTACTCCTGAGCGTACGGATATTGCTCGACTTCTGGA TATGGATGGCATTATAGTTGAAAAACAGCGTCGCCTTGCAACACTACTAGGTCTTCA AGCCCCACCGACACGAATTGGCCTTATTAATGATATGGTCAGATTTAATGTACTACA ATATGTTGTCCCAGAAGTGAAAGACCTTTACAATTGGCTTGAAGTAGAATTTAACCC 
ATTAAAACTCTGTGAGCGAGTCACAAAGGTTCTAAATTGGGTTAGGGAACAACCTGA AAAGGAACCGGAATTGCAGCAGTATGTGCCACAACTGCAAAACAACACCATCCTCCG CCTTCTGCAGCAGGTGTCACAGATTTATCAGAGCATTGAGTTTTCTCGTTTGACTTCTT TGGTTCCTTTTGTTGATGCTTTCCAACTGGAACGGGCCATAGTAGATGCAGCCAGGCA TTGCGACTTGCAGGTTCGTATTGATCACACTTCTCGGACCCTGAGTTTTGGATCTGAT TTGAATTATGCTACTCGAGAAGATGCTCCGATTGGTCCTCATTTGCAAAGCATGCCTT CAGAGCAGATAAGAAACCAGCTGACAGCCATGTCCTCAGTACTTGCAAAAGCACTTG AAGTCATTAAACCAGCTCATATACTGCAAGAGAAAGAAGAACAGCATCAGTTGGCTG TCACTGCATACCTTAAAAATTCACGAAAAGAGCACCAGCGGATCCTGGCTCGCCGCC AGACAATTGAGGAGAGAAAAGAGCGCCTTGAGAGTCTGAATATTCAGCGTGAGAAA GAAGAATTGGAACAGAGGGAAGCTGAACTCCAGAAAGTGCGGAAGGCTGAGGAAG AGAGGCTGCGCCAGGAAGCAAAGGAGAGAGAGAAGGAGCGTATCTTACAGGAACAT GAACAAATCAAAAAGAAAACTGTCCGAGAGCGTTTGGAGCAGATCAAGAAAACAGA ACTGGGTGCCAAAGCATTCAAAGATATTGATATTGAAGACCTTGAGGAATTGGATCC AGATTTTATCATGGCTAAACAGGTTGAACAACTGGAGAAAGAAAAGAAAGAACTTC AAGAACGCCTAAAGAATCAAGAAAAGAAGATTGACTATTTTGAAAGAGCCAAACGT TTGGAAGAAATTCCTTTGATAAAGAGCGCTTACGAGGAACAGAGAATTAAAGACATG GATCTGTGGGAGCAACAAGAGGAAGAAAGAATTACTACAATGCAGCTAGAACGTGA AAAGGCTCTTGAACATAAGAATCGAATGTCACGAATGCTTGAAGACAGAGATTTATT CGTAATGCGACTCAAAGCTGCACGGCAGTCTGTTTATGAGGAAAAACTTAAACAGTT TGAAGAGCGATTAGCAGAAGAAAGGCATAATCGATTGGAAGAACGGAAAAGGCAGC GTAAAGAAGAACGCAGGATAACATACTATAGAGAAAAAGAAGAGGAGGAGCAGAG AAGGGCAGAAGAACAAATGCTAAAAGAGCGGGAAGAGAGAGAGCGCGCCGAACGA GCAAAACGCGAGGAAGAGCTACGAGAGTATCAGGAGCGGGTGAAGAAATTAGAAG AAGTGGAAAGGAAAAAACGCCAAAGGGAGTTGGAAATTGAAGAACGAGAACGGCG 
TAGAGAGGAAGAGAGAAGACTTGGCGATAGTTCCCTTTCTAGAAAGGACTCTCGTTG GGGAGATAGAGATTCAGAAGGCACCTGGAGAAAAGGACCTGAAGCAGATTCTGAGT GGAGAAGAGGCCCGCCAGAGAAGGAGTGGAGACGTGGAGAAGGGCGAGATGAGGA CAGGTCTCATAGAAGAGATGAAGAGCGGCCCCGGCGTCTGGGGGATGATGAAGATA GAGAGCCCTCTCTTAGACCAGACGATGATCGGGTTCCCCGGCGTGGCATGGATGATG ACAGAGGCCCTAGACGTGGTCCTGAGGAAGATAGGTTCTCTCGTCGTGGGGCAGACG ATGACCGGCCTTCCTGGCGTAACACAGATGATGACAGGCCTCCCAGACGAATTGCCG ATGAAGACAGGGGAAACTGGCGTCATGCGGATGATGACAGACCACCTAGACGAGGA CTGGATGAGGACAGAGGAAGCTGGCGAACAGCTGATGAGGACAGAGGACCAAGACG TGGGATGGATGATGACCGGGGGCCGAGGCGAGGAGGCGCTGATGATGAGCGATCAT CCTGGCGTAATGCTGATGATGACCGGGGTCCCAGGCGAGGGTTGGATGATGATCGGG GTCCCAGGCGAGGCATGGATGATGACCGGGGTCCCAGGCGAGGCATGGATGATGAC CGGGGTCCCAGGCGAGGCATGGATGATGACCGGGGTCCCAGGCGAGGGTTGGATGA TGATCGAGGACCTTGGAGGAACGCCGATGATGACAGAATTCCCAGGCGTGGTGCAG AGGATGACAGGGGCCCTTGGAGAAACATGGATGATGATCGCCTTTCAAGACGTGCTG ATGATGATCGGTTTCCCAGACGGGGTGATGACTCAAGACCTGGTCCTTGGAGACCAT TAGTCAAGCCAGGTGGATGGAGAGAGAAAGAAAAAGCCAGAGAGGAGAGCTGGGG TCCACCTCGAGAATCAAGGCCATCAGAAGAACGTGAATGGGACAGAGAAAAAGAAA GGGACAGAGATAATCAAGATCGGGAGGAGAATGACAAGGACCCTGAGAGAGAAAG GGACAGAGAGAGAGATGTGGATCGAGAGGATCGCTTCAGAAGACCTAGGGATGAAG GTGGCTGGAGAAGAGGACCAGCTGAGGAATCTTCAAGCTGGAGAGACTCAAGTCGC CGGGACGATAGGGATAGGGATGACCGTCGCCGTGAGAGGGATGACCGGCGTGATCT AAGAGAAAGACGAGATCTAAGAGACGACAGGGACCGAAGAGGACCTCCACTCAGAT CAGAACGTGAAGAAGTAAGTTCTTGGAGACGTGCTGATGACAGGAAAGATGACCGG GTGGAAGAGCGGGACCCTCCTCGTCGAGTTCCTCCCCCAGCTCTTTCAAGAGACCGA 
GAAAGAGACCGAGACCGAGAAAGAGAAGGTGAAAAAGAGAAGGCCTCATGGAGAG

CTGAGAAAGATAGGGAATCTCTCCGTCGTACTAAAAATGAGACTGATGAAGATGGAT

GGACCACAGTACGACGTTAG

EIF3A HLH* - AAAAGTAAAAA mutated to AACAGTGAAGA

EIF3A shRNA target - GCGCCTTGAGAGTCTGAATAT

EIF3A shRNA target (mutated) - GCGACTAGAAAGCCTAAACAT

EIF3A $\mathrm{HLH}^{*}$ fasta file sequence (for building Bowtie2 indices) -

ATGTTCTTTATGATGTTATGAACAGTGAAGACATAGAACATGGCAAAAG

HCV IRES (start codon indicated in red)

CTCCCCTGTGAGGAACTACTGTCTTCACGCAGAAAGCGTCTAGCCATGGCGTTAGTAT

GAGTGTCGTGCAGCCTCCAGGACCCCCCCTCCCGGGAGAGCCATAGTGGTCTGCGGA

ACCGGTGAGTACACCGGAATTGCCAGGACGACCGGGTCCTTTCTTGGATTAACCCGC

TCAATGCCTGGAGATTTGGGCGTGCCCCCGCGAGACTGCTAGCCGAGTAGTGTTGGG

TCGCGAAAGGCCTTGTGGTACTGCCTGATAGGGTGCTTGCGAGTGCCCCGGGAGGTC

TCGTAGACCGTGCATCATGAGCACAAATCCT

MYC 5'-UTR

GACCCCCGAGCTGTGCTGCTCGCGGCCGCCACCGCCGGGCCCCGGCCGTCCCTGGCT

CCССтCCTGCCTCGAGAAGGGCAGGGCTTCTCAGAGGCTTGGCGGGAAAAAGAACG

GAGGGAGGGATCGCGCTGAGTATAAAAGCCGGTTTTCGGGGCTTTATCTAACTCGCT

GTAGTAATTCCAGCGAGAGGCAGAGGGAGCGAGCGGGCGGCCGGCTAGGGTGGAAG

AGCCGGGCGAGCAGAGCTGCGCTGCGGGCGTCCTGGGAAGGGAGATCCGGAGCGAA

TAGGGGGCTTCGCCTCTGGCCCAGCCCTCCCGCTGATCCCCCAGCCAGCGGTCCGCA

ACCCTTGCCGCATCCACGAAACTTTGCCCATAGCAGCGGGCGGGCACTTTGCACTGG 
AACTTACAACACCCGAGCAAGGACGCGACTCTCCCGACGCGGGGAGGCTATTCTGCC CATTTGGGGACACTTCCCCGCCGCTGCCAGGACCCGCTTCTCTGAAAGGCTCTCCTTG CAGCTGCTTAGACG

ATF4 5'-UTR (red ATG, uORF start codon)

TTTCTACTTTGCCCGCCCACAGATGTAGTTTTCTCTGCGCGTGTGCGTTTTCCCTCCTC CCCGCCCTCAGGGTCCACGGCCACCATGGCGTATTAGGGGCAGCAGTGCCTGCGGCA GCATTGGCCTTTGCAGCGGCGGCAGCAGCACCAGGCTCTGCAGCGGCAACCCCCAGC GGCTTAAGCCATGGCGCTTCTCACGGCATTCAGCAGCAGCGTTGCTGTAACCGACAA AGACACCTTCGAATTAAGCACATTCCTCGATTCCAGCAAAGCACCGCAACA ATF4 $\triangle \mathrm{uORF} 1$ - first ATG mutated to AGG ATF4 $\triangle \mathrm{uORF} 2$ - second ATG mutated to AGG

$H B B$ 5'-UTR

ACATTTGCTTCTGACACAACTGTGTTCACTAGCAACCTCAAACAGACACC 
bioRxiv preprint doi: https://doi.org/10.1101/354399; this version posted June 22, 2018. The copyright holder for this preprint (which was not certified by peer review) is the author/funder. All rights reserved. No reuse allowed without permission.

\section{Supplementary Figures}

A
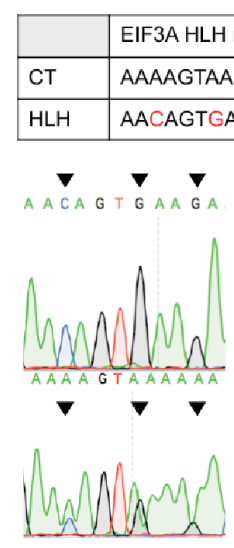

B

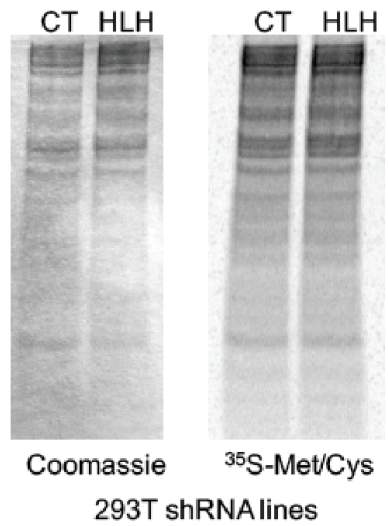

CT HLH

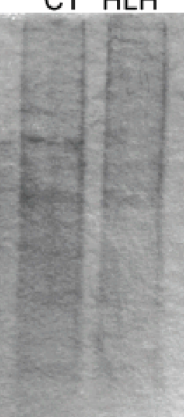

Coomassie CRISPRi 293T lines

C CT vs HLH Transcriptional Changes

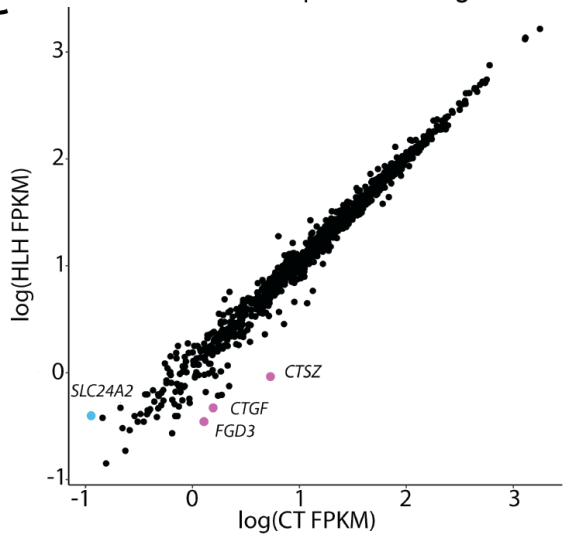

D

RS Fold Change vs FDR corrected p-value

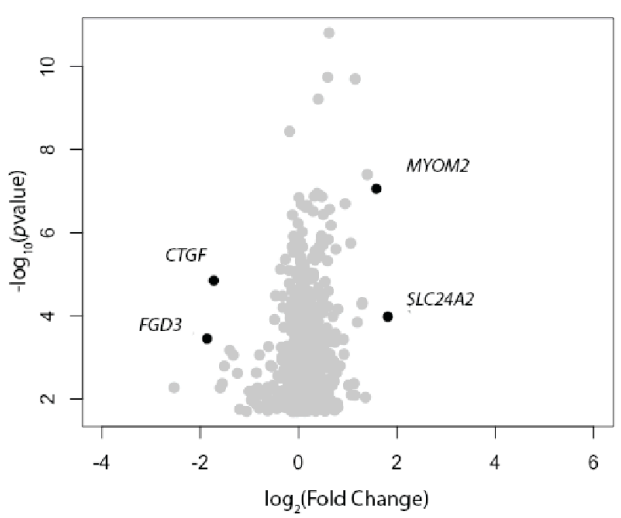

$E$
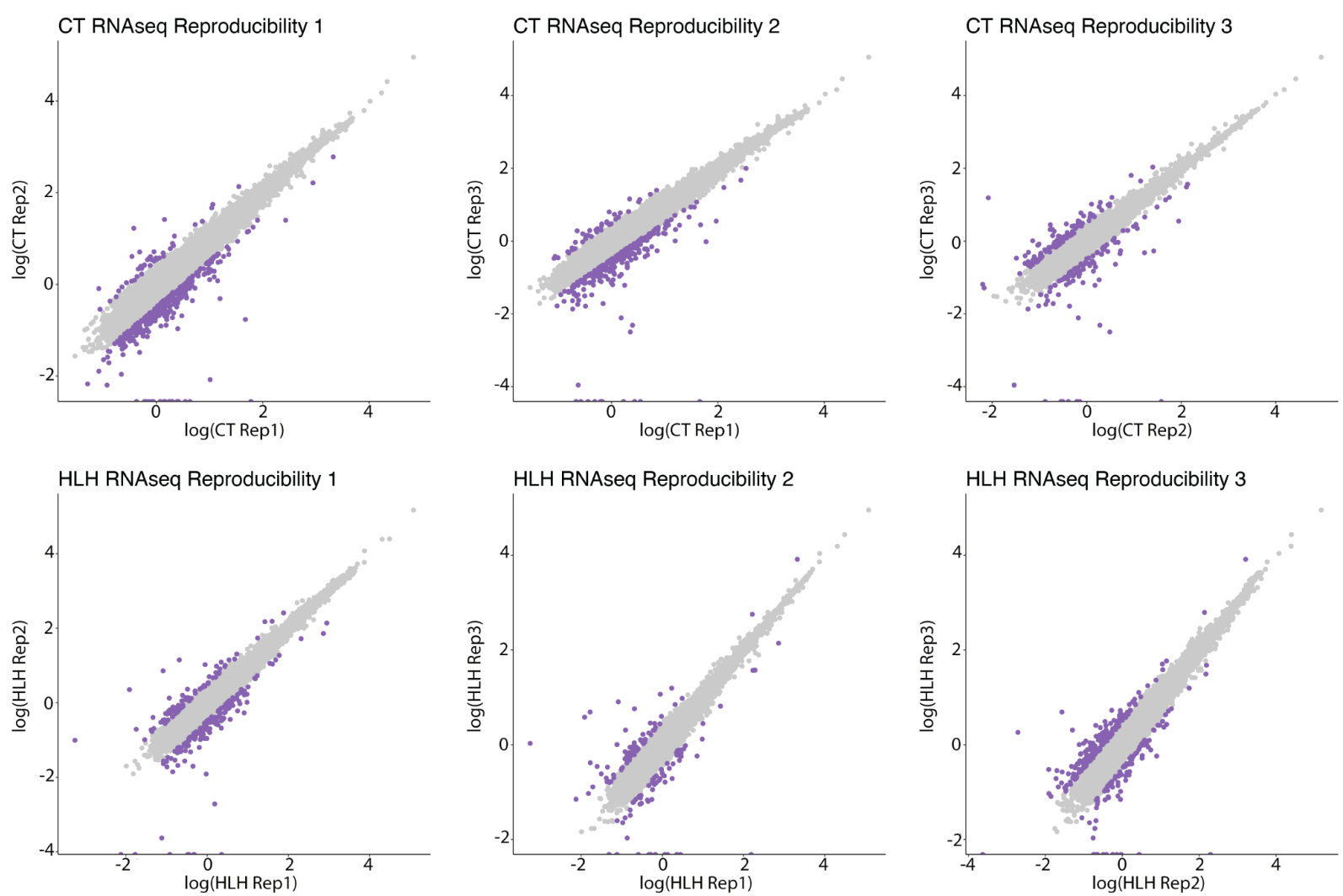
Fig. S1. Phenotypic and bioinformatic analyses of the shRNA lentiviral 293T cell lines. A.

Transient transfection of CT and HLH* (HLH) lentiviral vectors into HEK293T cells was analyzed by PCR-amplification and sequencing of the HLH mutation region (left) and western blotting for MYC protein levels (right). Levels of protein normalized to ACTB control given below gels. The arrows indicate the location of nucleotide base signal reflecting the HLH* mutations on the chromatogram. B. ${ }^{35}$ S-Met/Cys metabolic labeling of the shRNA lentiviral HEK294T and CRISPRi cell lines. Total protein is stained by Coommassie (left). C. Scatter plot of CT versus HLH* (HLH) transcriptional changes using average FPKM values of three biological replicates, showing transcripts meeting a $p$-value cutoff of 0.01 . Three transcripts in pink are downregulated $>3 x$, and one transcript in blue is upregulated $>3 x$. D. Volcano plots of transcriptional fold change against FDR-corrected $p$-value, showing transcripts meeting an FDR cutoff of 0.05 . Four transcripts in black show a $>2 x$ change in expression. E. RNAseq reproducibility scatter plots of CT and HLH biological replicates, showing non-zero transcripts used for statistical analysis. Highlighted in purple are transcripts with a $>3 x$ change in expression. 


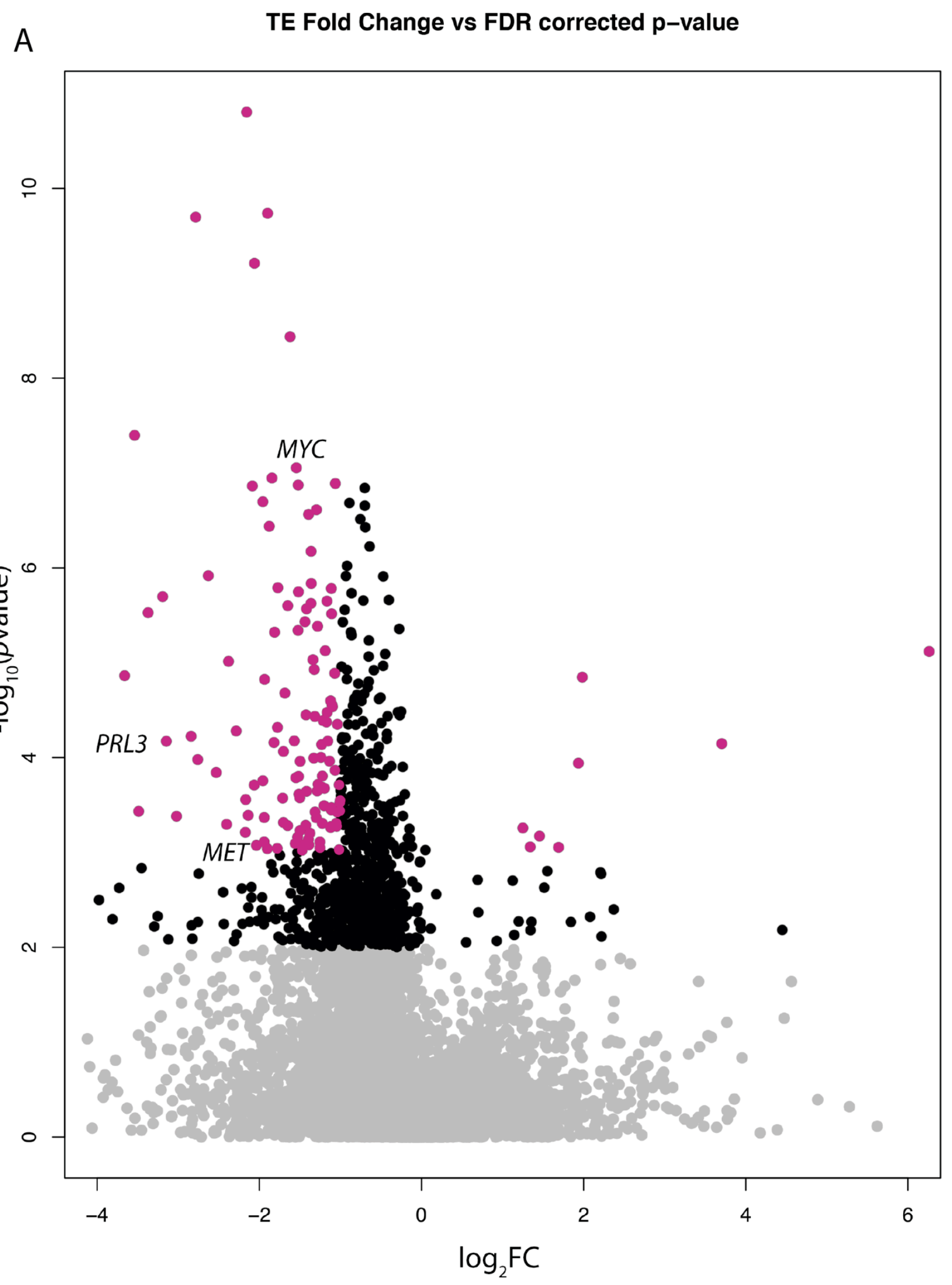



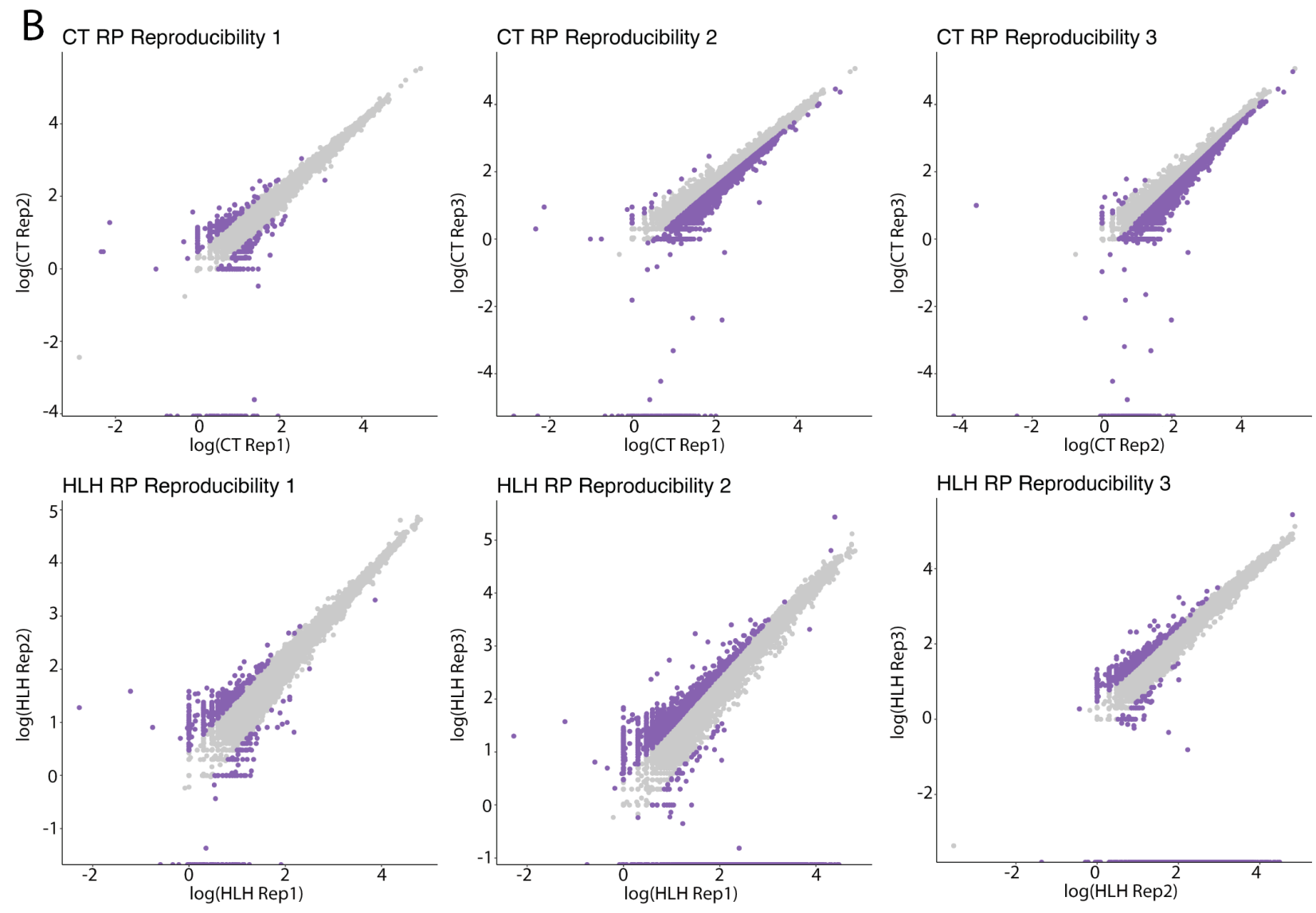

Fig. S2. Volcano plot of translational efficiency fold change against FDR corrected $p$-value.

A. Transcripts meeting a p-value cutoff of 0.01 in black. Out of those, transcripts with $>3 x$ fold change are highlighted in magenta. B. Ribosome profiling reproducibility scatter plots of CT and HLH* (HLH) biological replicates, showing non-zero transcripts used for statistical analysis.

Highlighted in purple are transcripts with a $>3 \mathrm{x}$ change in expression. 


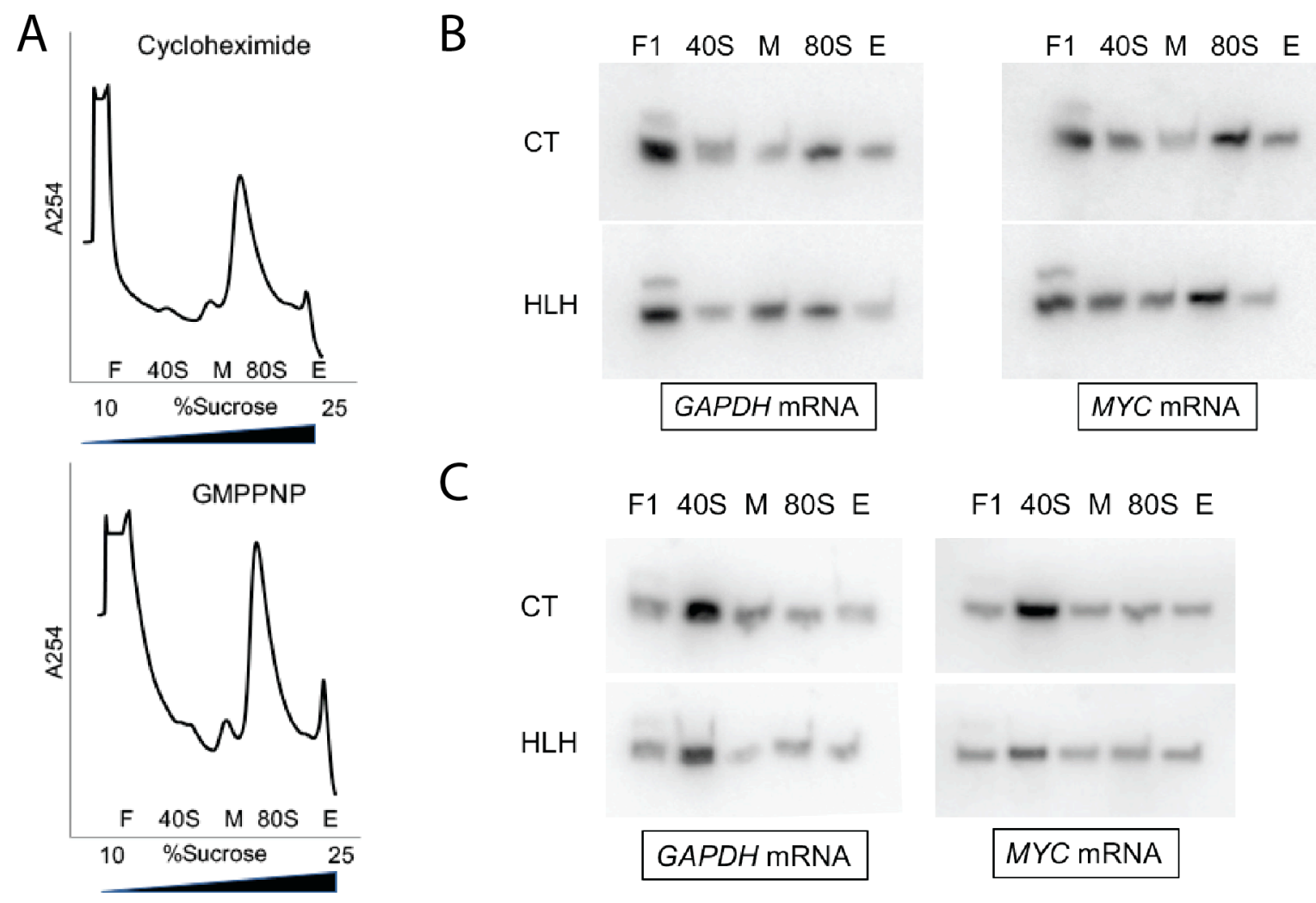

Fig. S3. Met-tRNA $A_{i}$ distribution in sucrose gradient fractions of in vitro translation

reactions. A. Sucrose gradient profiles of cycloheximide (top) and GMPPNP (bottom) stalled in vitro translation reactions fractionated on $10-25 \%$ sucrose gradients. B. Northern blotting of MettRNA $_{i}$ in the presence of cycloheximide. $\mathbf{C}$. Northern blotting of $\mathrm{tRNA}_{\mathrm{i}}$ in the presence of GMPPNP. 
A

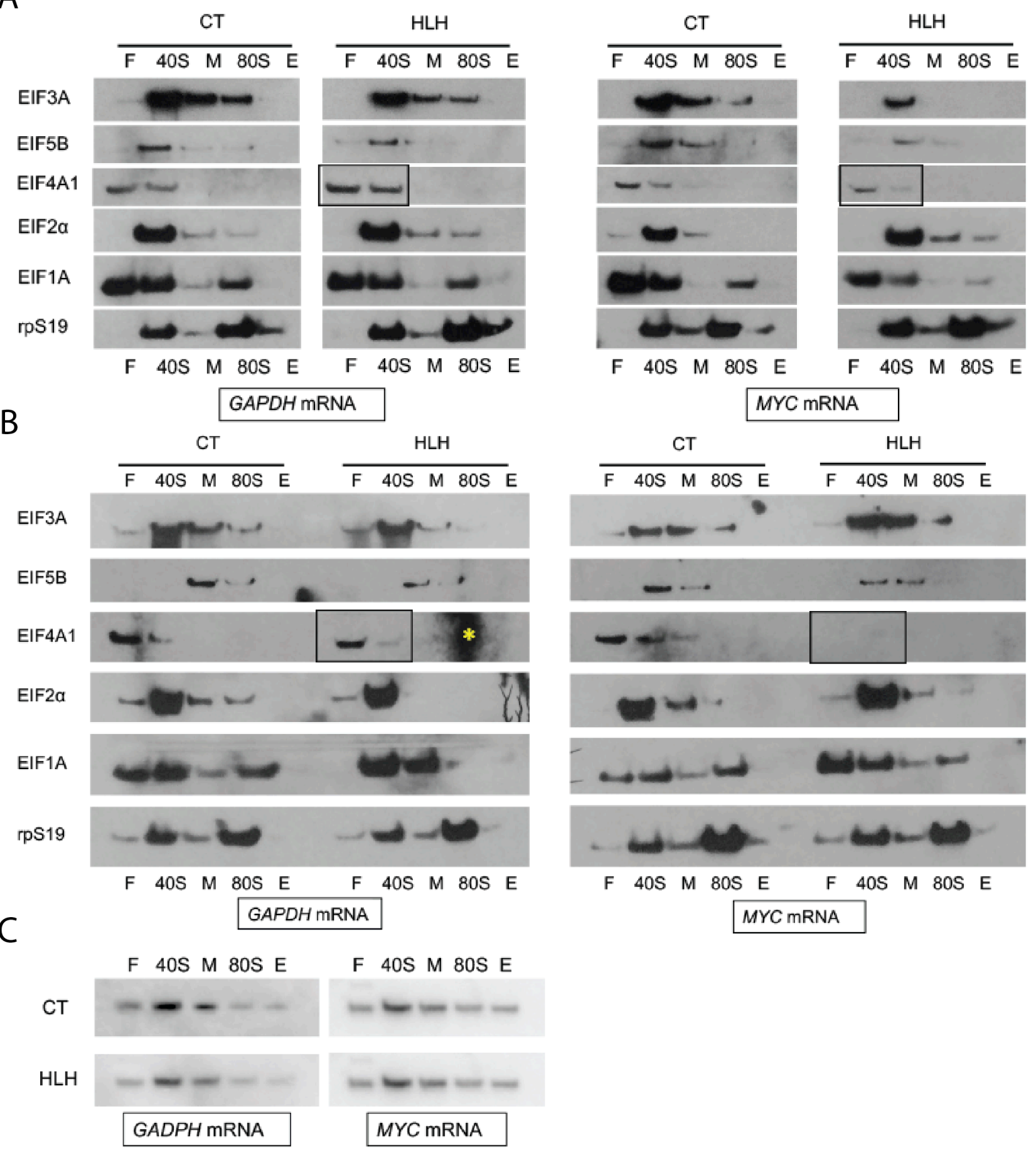

Fig. S4. Initiation factor distribution in GMPPNP and GMPPNP/RocA stalled in vitro

translation reactions. A. Western blot analysis of initiation factors in GMPPNP stalled in vitro translation reactions resolved by sucrose gradient fractionation, expanded from Fig. 2 to include additional factors. B. Western blot analysis of initiation factors in GMPPNP/RocA stalled in vitro translation reactions, expanded from Fig. 3 in include additional initiation factors. Boxes indicate 
fractions of interest for EIF4A1 levels. Yellow asterisk indicates background signal in gel, does not interfere with initiation factor distribution analysis. C. Northern blot analysis of Met-tRNA distribution in GMPPNP/RocA stalled in vitro translation reactions. 
A

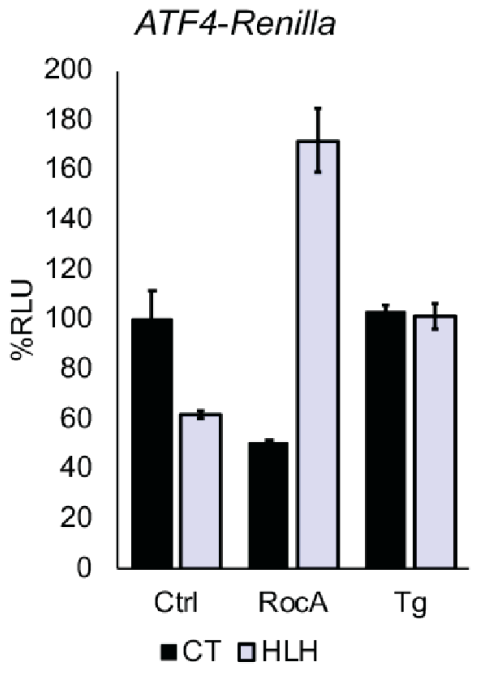

C

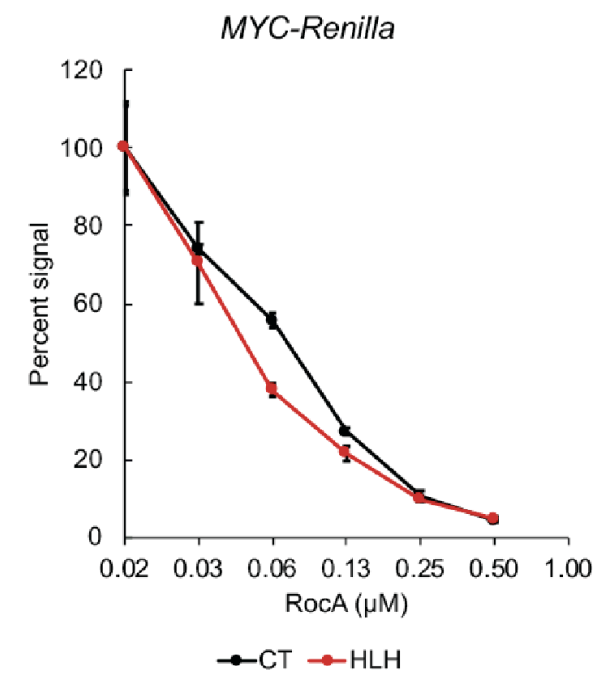

B

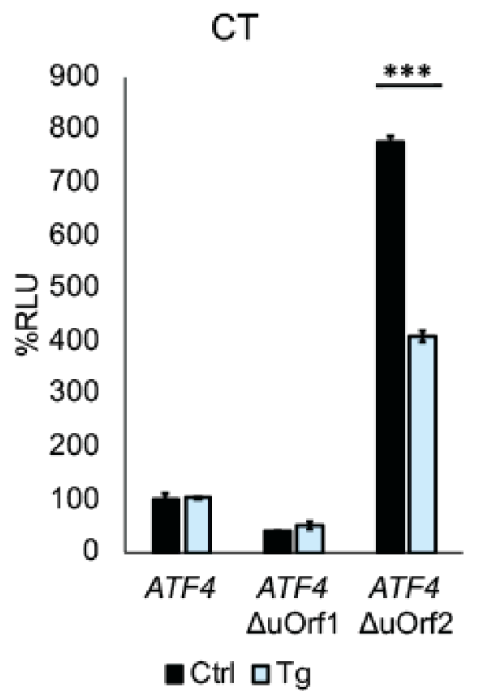

HLH

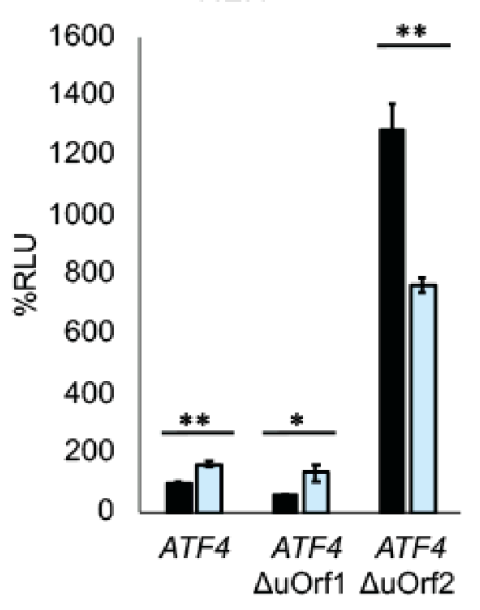

$\mathbf{c}$ trl $\square \mathrm{Tg}$

Fig S5. MYC 5'-UTR response to RocA and ATF4 5'-UTR response to thapsigargin. A. Live cell transfections of mRNAs containing the ATF4 5'-UTR fused to the Renilla luciferase ORF in the presence of thapsigargin and RocA. Relative Luciferase Units (RLU) percentage was normalized to internal HBB 5'-UTR-Firefly luciferase mRNA control signal. B. Live cell transfections of ATF4 uORF variant 5'-UTRs (WT, $\triangle \mathrm{uORF} 1, \Delta \mathrm{uORF} 2$ ) fused to Renilla mRNAs in the presence of thapsigargin. Control samples are identical to those plotted in Fig. 3F. C. Live cell transfection of MYC 5'-UTR - Renilla luciferase mRNA was performed to observe the effect of RocA-induced stress. Relative Luciferase Units (RLU) percentage was normalized to internal HBB 5'-UTR-Firefly luciferase control signal. 
A

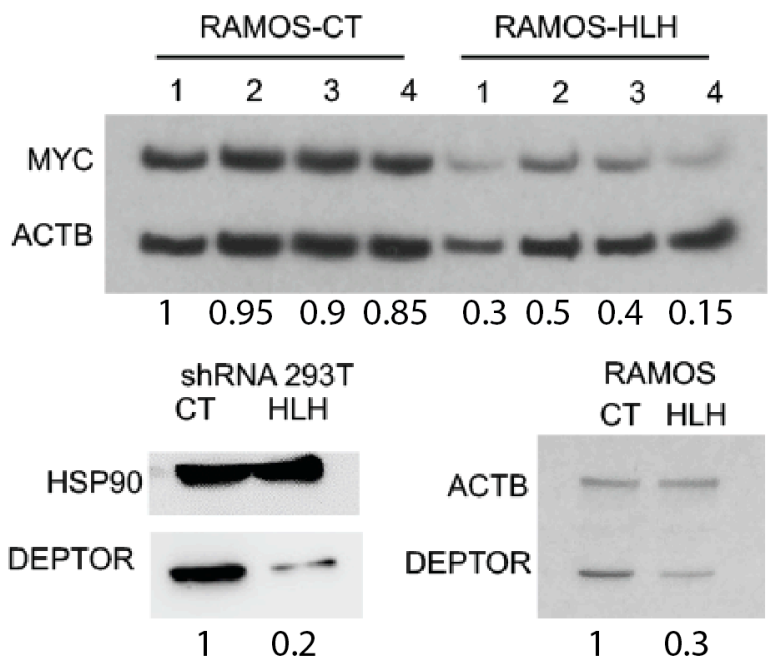

B

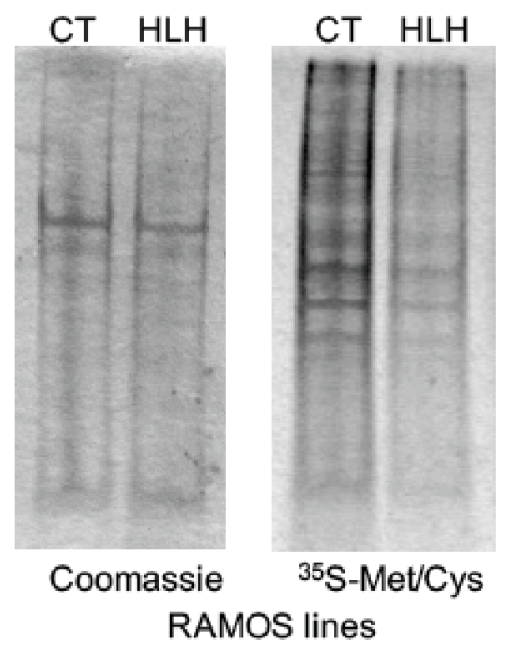

Fig. S6. Phenotypic analyses of Ramos Burkitt's lymphoma cell lines. A. Western blot validation of MYC suppression in Ramos HLH* cells. The numbers 1-4 represent separate cell lines transduced in parallel. Bottom panels show Western blot validation of additional cancerassociated negatively regulated transcript DEPTOR in HEK293T shRNA and Ramos shRNA cell lines. Levels of protein normalized to ACTB or HSP90 control given below gels. B. ${ }^{35}$ S-Met/Cys metabolic labeling of the CRISPRi and Ramos cell lines. Total protein is stained by Coommassie (left). 


\section{Supplementary Tables}

Included in a single Excel .xlsx file:

Table S1. RNAseq data (Supplementary Tables, tab 1, "RNAseq nonzero”)

Table S2. Ribosome profiling data (Supplementary Tables, tab 2, "RP (RSnonzero match)")

Table S3. Babel statistical analysis of RNAseq and ribosome profiling data (Supplementary

Tables, tab 3, "Babel_TE_FC”)

Table S4. Statistically significant transcripts from Babel analysis (Supplementary Tables, tab 4, "Babel_p<0.01") 